\title{
Phenolic profiles of Lauraceae plant species endemic to Laurisilva forest: A chemotaxonomic survey
}

\author{
Eulogio J. Llorent-Martínez ${ }^{\mathrm{a}, *}$, Vítor Spínola ${ }^{\mathrm{b}}$, Paula C. Castilho ${ }^{\mathrm{b}}$ \\ a University of Castilla-La Mancha, Regional Institute for Applied Chemistry Research (IRICA), Ciudad Real 13071, Spain \\ b CQM - Centro de Química da Madeira, Universidade da Madeira, Campus da Penteada, 9020-105 Funchal, Portugal
}

\section{A R T I C L E I N F O}

\section{Keywords:}

Macaronesia

Lauraceae

Flavonoids

Procyanidins

Epicatechin

\begin{abstract}
A B S T R A C T
In this work, the phenolic composition of several trees endemic to Madeira archipelago (Portugal) was studied. Specifically, the leaves of the most relevant species of the Lauraceae family (Laurus novocanariensis, Apollonias barbujana, Ocotea foetens, and Persea indica) have been analyzed. The screening of the main phenolic compounds in their methanol extracts has been performed by high-performance liquid chromatography with electrospray ionization mass spectrometric detection (HPLC-ESI-MS ${ }^{n}$ ), identifying or tentatively characterizing almost 100 compounds, including a high number of proanthocyanidins (A- and B-type), which have been reported to present remarkable health benefits. Thirty-four compounds have been quantified, observing total individual phenolic contents (TIPCs) between 18.43 and $88.99 \mathrm{mg} \mathrm{g}^{-1}$ dry extract, with the lowest TIPC in $O$. foetens and the highest in A. barbujana.
\end{abstract}

\section{Introduction}

Madeira (Madeira archipelago, Portugal) laurel forest, Laurisilva, is a subtropical forest with a very rich bryophyte and vascular flora. It is well characterized from the botanical point of view, but little attention has been paid to the composition of most plant species, even though several species have been used for centuries in folk medicine (Rivera and Obón, 1995). Among the different compounds that are present in plants, phenolic compounds are of special interest to scientists, as they exhibit important biological activities, such as anti-oxidant, antimicrobial, anti-cancer and anti-mutagenic. Therefore, the characterization of these compounds, mainly uninvestigated in wild plants, is an important research field nowadays. The chemical and biological characterization of these plants may lead to promising sources of biologically active compounds.

In a previous study (Llorent-Martínez et al., 2015a), our research group established the phytochemical composition of the most important non-lauraceae trees of the Laurisilva forest (Olea europaea ssp. cerasiformis, Ilex perado ssp. perado, Clethra arborea, and Heberdenia excelsa). In this sense, the aim of the present work was to investigate the phenolic profile of selected plants from the Laurisilva belonging to the Lauraceae family: Laurus novocanariensis, Apollonias barbujana, Ocotea foetens, and Persea indica. Other species from the Lauraceae family have been reported to present health benefits, due to their phytochemical profile. For instance, cinnamon (Cinnamomum zeylanicum and C. cassia barks) has been the most studied species, which extracts are recognized by their high levels of procyanidins (Rao and Gan, 2014). The study of other non-edible Lauraceae species may lead to new sources of proanthocyanidins.

Laurus is a genus of evergreen trees belonging to the Lauraceae family, and three autochthonous species [Laurus nobilis L., Laurus azorica (Seub.) Franco and Laurus novocanariensis Rivas-Mart., Lousã, Fern. Prieto, E. Díaz, J.C. Costa \& C. Aguiar] are described in Portugal (Vinha et al., 2015). L. novocanariensis is the most abundant endemic laurel from the Madeira archipelago and can grow from 3 to $20 \mathrm{~m}$ tall, presenting aromatic, shiny dark-green foliage. It presents male and female flowers on separate plants (Press and Short, 1994); the latter produce ovoid berries $(1-1.5 \mathrm{~cm})$ black when ripe. These berries derive a fatty oil that has been used in traditional medicine to treat skin ailments (Viciolle et al., 2012). Additionally, leaves (non-edible) are used in traditional cuisine to flavor dishes (Vinha et al., 2015), and to prepare infusions to relieve common cold and as sudorific (Rivera and Obón, 1995). A previous investigation on L. novocanariensis leaves documented monomeric and oligomeric flavan-3-ols as major phenolics (Vinha et al., 2015).

Apollonias barbujana (Cav.) Bornm. is an evergreen tree of 3-30 m tall. The simple leaves are alternate, elliptic, entire and petiolate, 6-8 cm length and 3-4 cm width. This tree produces panicles of white six-stellate flowers from June to September. Its berries are ovoid, approximately $15 \mathrm{~mm}$ long and brownish-grey color when ripe (Press

\footnotetext{
* Corresponding author.

E-mail addresses: Eulogio.Llorent@uclm.es, ellorent@ujaen.es (E.J. Llorent-Martínez).
} 
and Short, 1994). This species has been used in folk medicine as diuretic, analgesic, antiulcerogenic, cytostatic, cardiotonic, expectorant, stomachic, sedative or carminative effects, and against rheumatic pain (Rivera and Obón, 1995). The total phenolics content and antioxidant activity of $A$. barbujana have been determined before (Tavares et al., 2010), but not the individual composition of phenolics.

Ocotea foetens (Aiton) Baill is an evergreen tree up to $30 \mathrm{~m}$ height. It usually grows with multiple trunks branched from its base. Leaves are 9-12 cm long and 3-5 cm wide, oblong lanceolate. Flowering season is from June to August. It produces hard and fleshy berries, dark-green, approximately $3-\mathrm{cm}$ long. Its leaves have been traditionally used to prepare infusions, used as antihypertensive. It has also been used to treat malignant diseases with poultices made of tender leaves and branchlets (Rivera and Obón, 1995). Its total phenolics content and antioxidant activity have been previously reported (Tavares et al., 2010), but its phytochemical profile remains unknown.

The genus Persea, belonging to the family Lauraceae, comprises about 190 species including its main species $P$. americana Miller (avocado) (Alvárez et al., 2016). Persea indica (L) Spreng is an evergreen tree up to $15-20 \mathrm{~m}$ tall, with a broad, rounded crown. It presents leaves without glands, $10-20 \times 3-8 \mathrm{~cm}$, elliptic. It grows berries of about $2 \mathrm{~cm}$, ellipsoid, bluish-black when ripe (Press and Short, 1994). The phytochemical composition of $P$. indica has been scarcely studied to date; only the presence of diterpenes has been reported (Alvárez et al., 2016).

The Laurisilva is part of UNESCO natural patrimony since 2000, and the forest is cleaned and thinned every year to prevent fire spread, and to improve the growth of healthy trees. Considering that the felled specimens and cut branches are discarded, the present work is part of a project aiming to validate traditional claims in order to promote applications of the discarded material from the forest.

\section{Experimental}

\subsection{Chemicals and reagents}

All reagents and standards were of analytical reagent (AR) grade unless stated otherwise. Caffeic acid ( $\geq 98 \%)$, diosmin $(\geq 90 \%)$, kaempferol $(\geq 97 \%)$, protocatechuic acid $(98 \%)$ and rutin $(\geq 95 \%)$ were purchased from Sigma-Aldrich (St. Louis, MO, USA). (+ )-catechin hydrated ( $>99 \%$ ), apigenin ( $\geq 99 \%$ ) and hesperidin $(\geq 98.5 \%)$ were obtained from Extrasynthese (Genay, France); and quercetin dihydrate ( $>99 \%$ ) from Riedel-de Haen. Stock solutions of $200 \mathrm{mg} / \mathrm{L}$ were prepared in ethanol (HPLC grade; Sigma). LC-MS grade acetonitrile $\left(\mathrm{CH}_{3} \mathrm{CN}, 99 \%\right)$ (LabScan; Dublin, Ireland) and ultrapure water (Milli-Q Waters purification system; Millipore; Milford, MA, USA) were used for the HPLC-MS analyses.

\subsection{Sample preparation and extraction of phenolic compounds}

Plant material was collected in different locations of Madeira Island (Portugal) as described in Table 1. Branches were cut from healthy plants in the mentioned locations.

Leaves were lyophilized to dryness (Alpha 1-2 LD Plus freeze dryer, CHRIST), ground to powder, and stored at $-20{ }^{\circ} \mathrm{C}$. Phenolic extraction followed a previous procedure (Spínola et al., 2014): $1 \mathrm{~g}$ of dry material was extracted with $25 \mathrm{~mL}$ of methanol in an ultrasonic bath (Bandelin Sonorex, Germany) at $35 \mathrm{kHz}$ and $200 \mathrm{~W}$ for $60 \mathrm{~min}$ (room temperature). Chlorophylls (which can interfere in the analyses) were removed by adsorption on activated charcoal and extracts were filtered and concentrated to dryness in a rotary evaporator (Buchi Rotavapor R-114; USA) at $40^{\circ} \mathrm{C}$. The resulting extracts were stored at $4{ }^{\circ} \mathrm{C}$ until further analysis.

\subsection{Chromatographic conditions}

The HPLC-DAD analysis was performed on a Dionex ultimate 3000 series instrument (Thermo Scientific Inc.) coupled to a binary pump, an autosampler and a column compartment (kept at $20^{\circ} \mathrm{C}$ ). Separation was carried out in a Phenomenex Gemini $\mathrm{C}_{18}$ column $(5 \mu \mathrm{m}, 250 \times 3.0 \mathrm{~mm}$ i.d.) using a mobile phase composed by $\mathrm{CH}_{3} \mathrm{CN}$ (A) and water/formic acid $(0.1 \%, v / v)$ at a flow rate of $0.4 \mathrm{~mL} \mathrm{~min}^{-1}$. The following gradient program was used: $20 \% \mathrm{~A}$ (0 $\mathrm{min}), 25 \% \mathrm{~A}(10 \mathrm{~min}), 25 \% \mathrm{~A}(20 \mathrm{~min})$, $50 \%$ A (40 min), $100 \%$ A (42-47 $\mathrm{min}$ ) and 20\% A (49-55 min). Sample solutions $\left(5 \mathrm{mg} \mathrm{mL}^{-1}\right.$ ) were prepared by dissolving the dried extract in the initial HPLC mobile phase; after filtration through $0.45 \mu \mathrm{m}$ PTFE membrane filters, $5 \mu \mathrm{L}$ was injected.

For HPLC-ESI-MS ${ }^{\mathrm{n}}$ analysis, a Bruker Esquire model 6000 ion trap mass spectrometer (Bremen, Germany) with an ESI source was used in hyphenation with the former described Dionex HPLC system. MS $^{\mathrm{n}}$ analysis was performed in negative and positive modes and scan range was set at $m / z 100-1000$ with a speed of $13,000 \mathrm{Da} / \mathrm{s}$. The ESI conditions were as follows: drying and nebulizer gas $\left(\mathrm{N}_{2}\right)$ flow rate and pressure, $10 \mathrm{~mL} \mathrm{~min}^{-1}$ and $50 \mathrm{psi}$; capillary temperature, $325^{\circ} \mathrm{C}$; capillary voltage, $4.5 \mathrm{keV}$; collision gas $(\mathrm{He})$ pressure and energy, $1 \times 10^{-5}$ mbar and $40 \mathrm{eV}$. The acquisition of $\mathrm{MS}^{\mathrm{n}}$ data was made in auto $\mathrm{MS}^{\mathrm{n}}$ mode, with an isolation width of $4.0 \mathrm{~m} / \mathrm{z}$, and a fragmentation amplitude of $1.0 \mathrm{~V}\left(\mathrm{MS}^{\mathrm{n}}\right.$ up to $\left.\mathrm{MS}^{4}\right)$. Esquire control software was used for the data acquisition and Data Analysis for processing.

\subsection{Quantification of polyphenols}

For this experiment, one polyphenol was selected as the standard for each group, and it was used to calculate individual concentrations by HPLC-DAD (Spínola et al., 2014). Caffeic and protocatechuic acids were used, respectively, for hydroxycinnamic and hydroxybenzoic acids determinations. Quercetin, (+)-catechin, hesperidin and apigenin were the standards used for flavonols, flavanols, flavanones and flavones, respectively. Stock standard solutions $\left(1000 \mathrm{mg} \mathrm{L}^{-1}\right)$ were prepared in methanol, and calibration curves were built by diluting the stock solutions with the initial mobile phase. Six concentrations (5-100 $\mathrm{mg} \mathrm{L}^{-1}$ ) were used for the calibration, plotting peak area versus concentration $\left(R^{2} \geq 0.967\right.$ in all cases). Total individual phenolic contents (TIPC) were defined as the sum of the quantified phenolic compounds.

\subsection{Statistical analysis}

All samples were assayed in triplicate and results are given as means \pm standard deviations. Data analysis was carried out by means

Table 1

List of analyzed species and nomenclature used.

\begin{tabular}{|c|c|c|c|c|c|}
\hline Species & Common name & & Collection Area & Collection data & Voucher number \\
\hline \multirow[t]{3}{*}{ L. novocanariensis } & Loureiro & L1 & Ribeiro Frio & March 2013 & MADJ 9677 \\
\hline & & $\mathrm{L} 2$ & Chão dos Louros & March 2013 & MADJ 11285 \\
\hline & & L3 & Ponta do Pargo & July 2013 & MADJ 14155 \\
\hline A. barbujana & Barbusano & $\mathrm{AJ}$ & Ribeiro Frio & March 2013 & MADJ 4765 \\
\hline O. foetens & Til & OF & Ribeiro Frio & March 2013 & MADJ 13159 \\
\hline P. indica & Vinhático & PI & Ribeiro Frio & March 2013 & MADJ 13157 \\
\hline
\end{tabular}



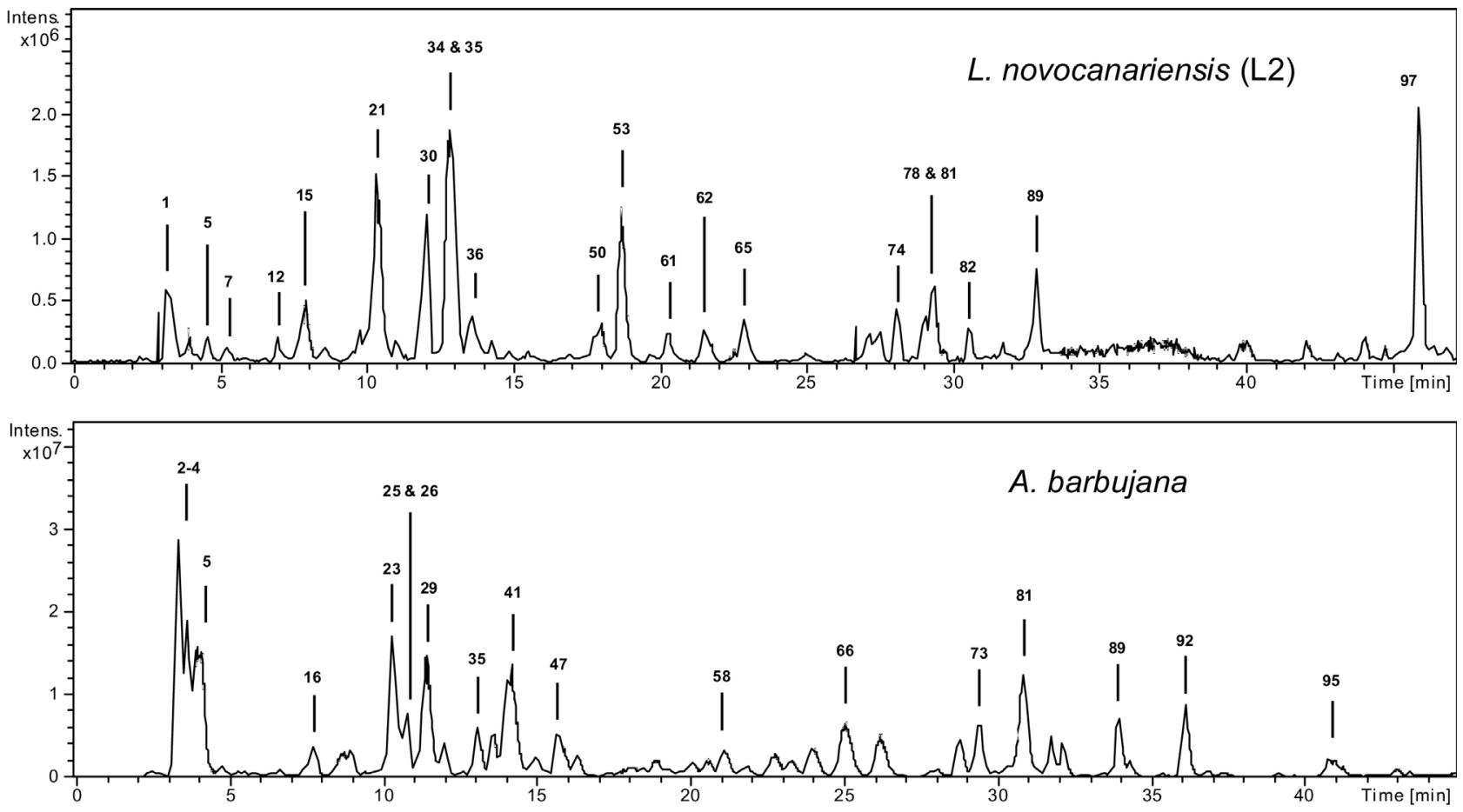

Fig. 1. HPLC-ESI/MS ${ }^{\mathrm{n}}$ base peak chromatograms (BPCs) of L. novocanariensis and A. barbujana methanol extracts (negative mode).

of a one-way ANOVA with Tukeys post-hoc test using SPSS for Windows, IBM SPSS Statistics 20 (SPSS, Inc., USA). A value of $p<0.05$ was considered statistically significant. Principal component analysis (PCA) was applied to the concentrations of polyphenols determined in different Lauraceae plants.

\section{Results and discussion}

\subsection{HPLC-ESI-MS}

The analysis of the phenolic composition in the extracts of the leaves from the different plants was carried out by HPLC-ESI-MS ${ }^{\mathrm{n}}$ using negative and positive ionization modes. Three independent assays were carried out for each sample, obtaining similar data concerning the nature and relative intensities of the fragments. The base peak chromatograms of the methanol extracts are shown in Figs. 1 and 2.

The initial step for the characterization of the compounds consisted in the determination of the molecular weights. With this purpose, the negative ionization mode was used. The base peak usually corresponded to the deprotonated molecular ion $[\mathrm{M}-\mathrm{H}]^{-}$, although several formate adducts $\left([\mathrm{M}-\mathrm{H}+\mathrm{HCOOH}]^{-}\right)$were also detected. For the identification of the flavonoid glycosides, the mass spectra of the aglycones were compared with analytical standards when available (apigenin, kaempferol and quercetin) and the chemical nature of the sugars was determined by the neutral losses observed. Rutin and diosmin were identified by comparison with analytical standards. When reference compounds were not available, the tentative characterization was carried out by comparison of the experimental mass spectra with data from scientific literature.

The positive ionization mode was used for confirmation of the identifications. Compounds were numbered in all the chromatograms by their order of elution, keeping the same numbering in the different plants. A total number of 97 compounds were characterized, distributed among the different species analyzed. The tentative characterization of the detected compounds is shown in Tables 2 and 3, and the discussion of the characterization is explained in the following sub-sections.

\subsubsection{Phenolic acids}

Compound 10, $[\mathrm{M}-\mathrm{H}]^{-}$at $m / z 361$, exhibited its $\mathrm{MS}^{2}$ base peak at $\mathrm{m} / \mathrm{z} 163$, which was identified as coumaric acid due to the $163 \rightarrow 119$ transition (Gruz et al., 2008). Hence, it was characterized as a coumaric acid derivative.

Compound 14 displayed an $[\mathrm{M}-\mathrm{H}]^{-}$ion at $m / z 353, \mathrm{MS}^{2}$ base peak ion at $m / z 191$ and a relatively intense fragment ion at $m / z 179$. According to Clifford et al. (2003), it was identified as 3-O-caffeoylquinic acid.

Compounds 19 and 35 presented $[\mathrm{M}-\mathrm{H}]^{-}$ions at $m / z 289$ and identical fragmentation patterns. Considering that catechin elutes before epicatechin in reversed-phase HPLC, 19 and 35 were identified as catechin and epicatechin, respectively (Stöggl et al., 2004).

Compound 25 exhibited the deprotonated molecular ion at $m / z 337$, and its $\mathrm{MS}^{2}$ base peak at $m / z$ 163. Considering bibliographic data (Clifford et al., 2003), it was characterized as 3-p-coumaroylquinic acid.

Compound 35 was identified as epicatechin as described before. Compound 42, with deprotonated molecular ion at $m / z$ 647, presented an $\mathrm{MS}^{2}$ base peak ion at $m / z 289$, and $\mathrm{MS}^{3}$ [647 $\rightarrow 289$ ] fragment ions at $m / z 245,205$ and 203, so it was tentatively characterized as an (epi) catechin derivative.

Compound 79, with $[\mathrm{M}-\mathrm{H}]^{-}$at $m / z$ 551, displayed an $\mathrm{MS}^{2}$ fragment ion at $m / z 153$, which had its main fragment ion at $m / z$ 109. Hence, it was characterized as a dihydroxybenzoic acid derivative.

\subsubsection{Proanthocyanidins (PAs)}

Ten PAs were detected in extracts of $L$. novocanariensis. Compounds 15, 33, 36, 65 and 75 exhibited $[\mathrm{M}-\mathrm{H}]^{-}$at $m / z 575$, and their mass fragmentation profiles (Tables 2 and 3 ) were similar to those reported for PA dimers (A-type), probably (epi)catechin-(epi)catechin (Soong and Barlow, 2005; Tomás-Barberán et al., 2001). Compounds 21 and 62 exhibited the deprotonated molecular ion at $m / z 577$, and fragment ions at $m / z 451,425,407$, and 289 (Table 2), typical from B-type PA dimers (Kajdžanoska et al., 2010; Ruiz et al., 2005). The fragment ion at $\mathrm{m} / \mathrm{z} 289$ proceeds from the loss of an (epi)catechin unit, so they were characterized as (epi)catechin-(epi)catechin dimers (Fig. 3). Compounds 30 and 34, with $[\mathrm{M}-\mathrm{H}]^{-}$at $m / z 863$, were characterized as A-type trimers (epi)catechin-(epi)catechin-(epi)catechin, comparing 

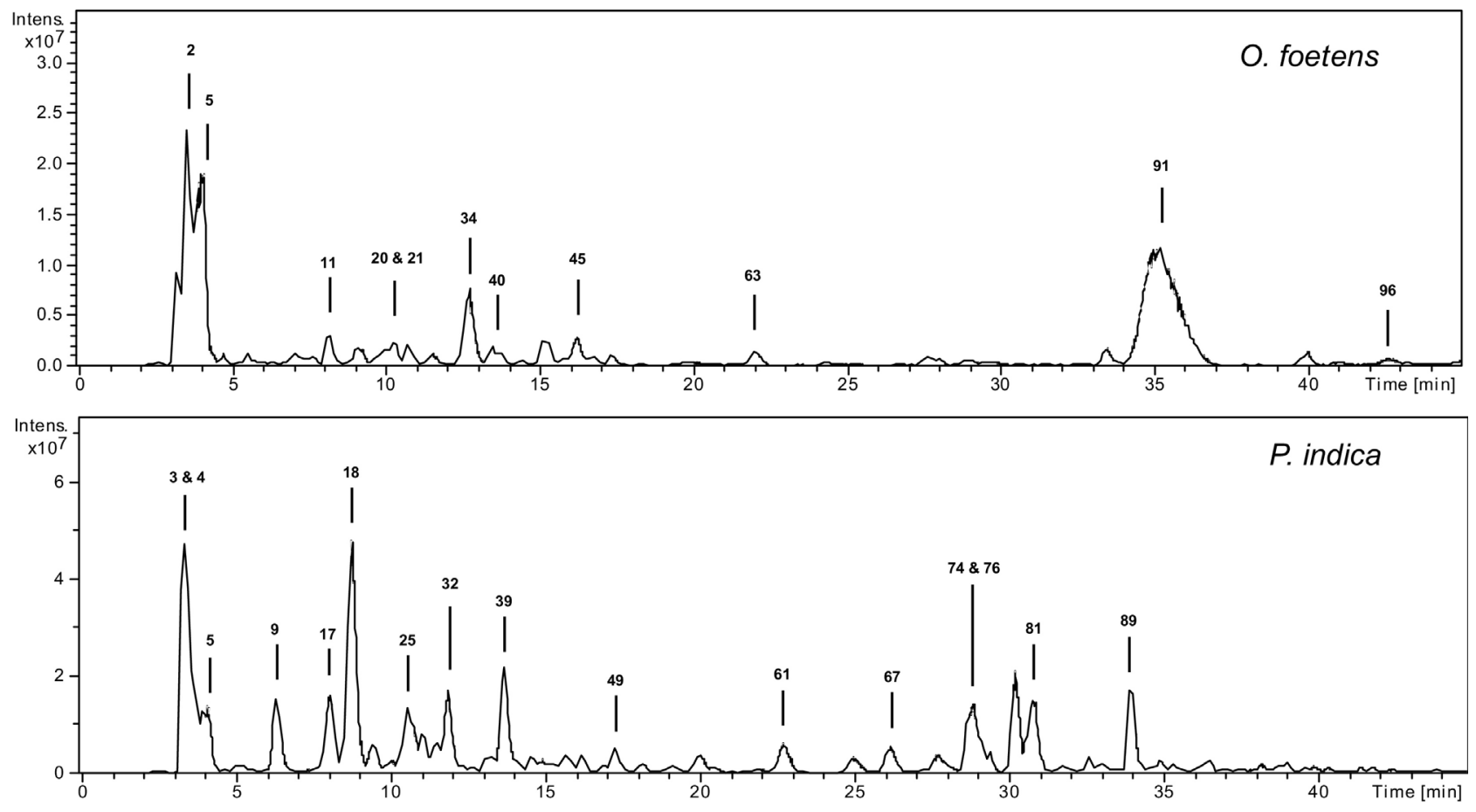

Fig. 2. HPLC-ESI/MS ${ }^{\mathrm{n}}$ base peak chromatograms (BPCs) of $O$. foetens and P. indica methanol extracts (negative mode).

the mass fragmentation (positive and negative ion modes) with data from bibliography (Määttä-Riihinen et al., 2005). 30 and 34 are PA trimers with one A-linkage, whereas compounds $\mathbf{8 3}$ was characterized as a trimer with two A-linkages (Appeldoorn et al., 2009).

Five proanthocynidins were detected in the analyzed extracts of $A$. barbujana. Compounds 43 and 65 exhibited $[\mathrm{M}-\mathrm{H}]^{-}$at $\mathrm{m} / \mathrm{z} 575$ and were characterized as PA dimers, probably (epi)catechin-(epi)catechin (A-type). Compounds 29 and 62, with $[\mathrm{M}-\mathrm{H}]^{-}$at $m / z 577$, were characterized as (epi)catechin-(epi)catechin dimers of the B-type (Kajdžanoska et al., 2010; Ruiz et al., 2005), as previously explained. Finally, compound $\mathbf{4 1}$ was characterized as an (epi)catechin-(epi) catechin-(epi)catechin trimer with one A-linkage.

Four PAs were detected in extracts of O. foetens, all of them consisting of (epi)catechin units. Compounds 21 and 29 were characterized as dimers $(m / z 577)$, whereas compounds 34 and 83 as trimers, with deprotonated molecular ions at $\mathrm{m} / \mathrm{z} 863$ and 861, respectively.

Six PAs were characterized in $P$. indica. Compounds 17, 21, 29, and 46 exhibited the deprotonated molecular ion at $m / z 577$, and fragment ions at $m / z 451,425,407$, and 289, typical from B-type PA dimers (Kajdžanoska et al., 2010; Ruiz et al., 2005). Compound 22, with $[\mathrm{M}-\mathrm{H}]^{-}$at $m / z$ 865, was characterized as an (epi)catechin-(epi) catechin-(epi)catechin trimer (B-type) (Kajdžanoska et al., 2010; Ruiz et al., 2005), whereas compound $34(\mathrm{~m} / \mathrm{z}$ at 863$)$ was identified as an A-type trimer (Määttä-Riihinen et al., 2005).

\subsubsection{Flavonoids}

High percentages - approximately $40 \%$ - of flavonoids were observed in all the analyzed extracts. The discussions in the following sub-sections are carried out grouping the compounds by their aglycones.

3.1.3.1 L. novocanariensis. Three apigenin derivatives were characterized. Compounds $\mathbf{5 0}$ and $\mathbf{5 3}$ were characterized as $C$-glycosil flavones, $O$-glycosylated on the sugar moiety, based on the characteristic peaks $[(\mathrm{M}-\mathrm{H})-164]$ and [aglycone $+41-18]$ at $\mathrm{m} /$ z 413 and 293. They were identified as rhamnosylvitexin and 2"-Orhamnosylvitexin, respectively, considering their elution order (Dou et al., 2007; Ferreres et al., 2007). Compound $\mathbf{5 4}$ was identified as apigenin-8-C-hexoside (vitexin), based on its fragment ions at $m / z 341$, 311 and 283 and the absence of a fragment ion at $m / z$ 413, which would indicate isovitexin (Gouveia and Castilho, 2013; Waridel et al., 2001).

Compounds 64 and 81 were quercetin-O-glycosides, exhibiting the aglycone at $m / z$ 301. 64 and 81 suffered neutral losses of hexoside (162 Da) and deoxyhexoside (146 Da), respectively.

Five kaempferol-O-glycosides were characterized, showing the aglycone at $m / z 285$. Compounds 72, 74, 82 and 89 suffered neutral losses of 308, 162, 132 and $146 \mathrm{Da}$, respectively, and were characterized as kaempferol $O$-rutinoside, $O$-hexoside, $O$-pentoside, and $O$ coumaroyl, respectively. Compound 97 could not be fully identified and was characterized as a kaempferol derivative.

Finally, compounds 78 and 85 corresponded to isorhamnetin derivatives, with the aglycone at $m / z 315$ and typical fragment ion at $\mathrm{m} / \mathrm{z}$ 300. Compound 78 was characterized as isorhamnetin- $O$-hexoside, whereas compound 85 corresponded to isorhamnetin-O-rutinoside or isorhamnetin-O-neohesperidoside (Bakr and El Bishbishy, 2016).

3.1.3.2 Apollonias barbujana. Nine quercetin derivatives were characterized (aglycone at $\mathrm{m} / \mathrm{z} 301$ ). Compound 47 suffered two neutral lossses of $162 \mathrm{Da}(625 \rightarrow 463$ and $463 \rightarrow 301)$, and was assigned to quercetin- $O$-dihexoside. Compound $\mathbf{5 8}$ presented the loss of hexoside + pentoside units. Compounds 64 and 66 corresponded to quercetin- $O$-hexoside isomers. Compounds $\mathbf{7 3}$ and $\mathbf{8 1}$ suffered neutral losses of 132 and $146 \mathrm{Da}$, respectively, and were characterized as quercetin- $O$-pentoside and quercetin-O-deoxyhexoside. Compound $\mathbf{6 8}$ was characterized as quercetin-O-xylo-pentoside (Sánchez-Rabaneda et al., 2004). Two compounds were identified as quercetin diglycosides, presenting the same $[\mathrm{M}-\mathrm{H}]^{-}$, together with the common neutral loss of $308 \mathrm{Da}(609 \rightarrow 301)$. Compound 61 was unambiguously identified as rutin after comparison with an analytical standard, whereas compound $\mathbf{7 1}$ was tentatively identified as quercetin- $O$-neohesperidoside, since rutinosides elute earlier than their corresponding neohesperidoside analogues (Abad-García et al., 2009).

Compound 55 presented the $[\mathrm{M}-\mathrm{H}]^{-}$at $m / z 521$. After the neutral 
Table 2

Characterization of the methanol extract of leaves from $L$. novocanariensis.

\begin{tabular}{|c|c|c|c|c|c|c|c|}
\hline No. & $\mathrm{t}_{R}(\mathrm{~min})$ & {$[\mathrm{M}-\mathrm{H}]^{-} m / z$} & $m / z$ (\% base peak) & Assigned identification & L1 & $\mathrm{L} 2$ & L3 \\
\hline 1 & 3.4 & 275 & $\begin{array}{l}\mathrm{MS}^{2}[275]: 239(100), 221(11), 179(9), 161(7), 149(33), 131(11) \\
119(5) \\
\operatorname{MS}^{3}[275 \rightarrow 239]: 179(100), 161(24), 149(93), 131(52), 119(36) \\
89(23)\end{array}$ & Saccharide derivative & $\checkmark$ & $\checkmark$ & $\checkmark$ \\
\hline 5 & 4.0 & 191 & $\operatorname{MS}^{2}$ [191]: $173(20), 111(100)$ & Quinic acid & $\checkmark$ & $\checkmark$ & $\checkmark$ \\
\hline 7 & 5.5 & 315 & $\begin{array}{l}\mathrm{MS}^{2}[315]: 153(100), 123(9) \\
\mathrm{MS}^{3}[315 \rightarrow 153]: 123(100)\end{array}$ & Hydroxytyrosol hexoside & & $\checkmark$ & \\
\hline 8 & 5.8 & 319 & $\mathrm{MS}^{2}$ [319]: $97(100)$ & Unknown & & & $\checkmark$ \\
\hline 10 & 6.6 & 361 & $\begin{array}{l}\operatorname{MS}^{2}[361]: 163(100) \\
\operatorname{MS}^{3}[361 \rightarrow 163]: 119(100)\end{array}$ & Coumaric acid derivative & & $\checkmark$ & \\
\hline 12 & 7.2 & 315 & $\begin{array}{l}\mathrm{MS}^{2}[315]: 153(100), 152(29) \\
\mathrm{MS}^{3}[315 \rightarrow 153]: 109 \text { (100), } 108(39)\end{array}$ & Dihydroxybenzoic acid-O-hexoside & $\checkmark$ & $\checkmark$ & $\checkmark$ \\
\hline 15 & 7.8 & 575 & $\mathrm{MS}^{2}$ [575]: 499 (41), 451 (54), 289 (35), 245 (100), 205 (17) & Proanthocyanidin dimer (A-type) & $\checkmark$ & $\checkmark$ & $\checkmark$ \\
\hline 19 & 9.5 & 289 & $\begin{array}{l}\mathrm{MS}^{2}[289]: 245(100), 205(27), 203(7), 179(26) \\
\mathrm{MS}^{3}[289 \rightarrow 245]: 227(8), 203(100)\end{array}$ & Catechin & & $\checkmark$ & \\
\hline 21 & 10.0 & 577 & $\operatorname{MS}^{2}$ [577]: 451 (12), 425 (94), 407 (100), 289 (14), 287 (10), 245 (14) & (Epi)catechin-(epi)catechin (B-type) & $\checkmark$ & $\checkmark$ & $\checkmark$ \\
\hline 28 & 10.9 & 431 & $\begin{array}{l}\operatorname{MS}^{2}[431]: 385(35), 179(100) \\
\operatorname{MS}^{3}[431 \rightarrow 179]: 161(6), 149(57), 143(65), 125(53), 119(6), 113 \\
(56), 89(100)\end{array}$ & Saccharide derivative (formate adduct) & $\checkmark$ & $\checkmark$ & $\checkmark$ \\
\hline 30 & 11.5 & $\begin{array}{l}863 \\
865(+)\end{array}$ & $\begin{array}{l}\mathrm{MS}^{2} \text { [863]: } 712(35), 711(100), 695(10), 693(46), 559(33), 451 \\
(17), 411(19), 289(10) \\
\mathrm{MS}^{3}[863 \rightarrow 711]: 694(64), 693(100), 559(77), 407(37) \\
\mathrm{MS}^{2}[865]: 713(72), 695(54), 533(100), 287(29) \\
\mathrm{MS}^{3}[865 \rightarrow 533]: 515(15), 407(42), 287(100)\end{array}$ & Proanthocyanidin trimer (A-type) & $\checkmark$ & $\checkmark$ & \\
\hline 33 & 12.4 & 575 & $\begin{array}{l}\mathrm{MS}^{2}[575]: 499(55), 490(55), 423(37), 407(16), 289(100) \\
\mathrm{MS}^{3}[575 \rightarrow 289]: 271(100), 245(54), 203(24)\end{array}$ & Proanthocyanidin dimer (A-type) & & $\checkmark$ & \\
\hline 34 & 12.6 & $865(+)$ & $\begin{array}{l}\mathrm{MS}^{2} \text { [863]: } 712(40), 711(100), 693(45), 559(30), 573(18), 451 \\
(20), 411(31), 289(9) \\
\mathrm{MS}^{3}[863 \rightarrow 711]: 693(100), 559(36), 541(21), 425(6), 407(23) \\
\mathrm{MS}^{2}[865]: 713(67), 695(49), 533(100), 287(19) \\
\mathrm{MS}^{3}[865 \rightarrow 533]: 515(10), 407(41), 287(100)\end{array}$ & Proanthocyanidin trimer (A-type) & $\checkmark$ & $\checkmark$ & $\checkmark$ \\
\hline 35 & 13.0 & 289 & $\operatorname{MS}^{2}$ [289]: 245 (100), 205 (31), 203 (9), 179 (11) & Epicatechin & $\checkmark$ & $\checkmark$ & $\checkmark$ \\
\hline 36 & 13.3 & 575 & $\mathrm{MS}^{2}$ [575]: 499 (100), 490 (95), 451 (83), 423 (43), 289 (80) & Proanthocyanidin dimer (A-type) & $\checkmark$ & $\checkmark$ & \\
\hline 50 & 17.6 & 577 & $\begin{array}{l}\mathrm{MS}^{2}[577]: 413(100), 293(76) \\
\mathrm{MS}^{3}[577 \rightarrow 413]: 293(100)\end{array}$ & Rhamnosylvitexin & $\checkmark$ & $\checkmark$ & $\checkmark$ \\
\hline 53 & 18.2 & 577 & $\operatorname{MS}^{2}$ [577]: 457 (37), 431 (34), 413 (28), 293 (100) & 2“-O-rhamnosylvitexin & $\checkmark$ & $\checkmark$ & $\checkmark$ \\
\hline 54 & 18.6 & 431 & $\begin{array}{l}\operatorname{MS}^{2}[431]: 341(10), 312(20), 311(100) \\
\left.\operatorname{MS}^{3} \text { [431 } \rightarrow 311\right]: 284(99), 283(100), 268(39)\end{array}$ & Apigenin-8-C-hexoside & & & $\checkmark$ \\
\hline 61 & 20.9 & 609 & $\begin{array}{l}\mathrm{MS}^{2}[609]: 301(100) \\
\operatorname{MS}^{3}[609 \rightarrow 301]: 271(21), 179(92), 151(100)\end{array}$ & Rutin & $\checkmark$ & $\checkmark$ & \\
\hline 62 & 21.5 & 577 & MS $^{2}$ [577]: 559 (23), 451 (9), 425 (100), 407 (54), 289 (28), 287 (14) & (Epi)catechin-(epi)catechin (B-type) & $\checkmark$ & $\checkmark$ & $\checkmark$ \\
\hline 64 & 23.0 & 463 & $\begin{array}{l}\mathrm{MS}^{2}[463]: 301(100), 300(18) \\
\mathrm{MS}^{3}[463 \rightarrow 301]: 179(100), 151(84)\end{array}$ & Quercetin-O-hexoside & $\checkmark$ & $\checkmark$ & \\
\hline 65 & 23.1 & 575 & $\operatorname{MS}^{2}$ [575]: 449 (45), 423 (100), 407 (7), 289 (6), 287 (10) & Proanthocyanidin dimer (A-type) & $\checkmark$ & $\checkmark$ & $\checkmark$ \\
\hline 72 & 28.3 & 593 & $\operatorname{MS}^{2}$ [593]: 285 (100), $163(56), 151(20)$ & Kaempferol-O-rutinoside & $\checkmark$ & & \\
\hline 74 & 28.7 & 447 & $\begin{array}{l}\mathrm{MS}^{2} \text { [447]: } 285(43), 284(100), 257(11), 255(39) \\
\operatorname{MS}^{3}[447 \rightarrow 285]: 255(100), 241(12), 151(35)\end{array}$ & Kaempferol-O-hexoside & $\checkmark$ & $\checkmark$ & $\checkmark$ \\
\hline 75 & 28.7 & 575 & $\mathrm{MS}^{2}$ [575]: 449 (49), $423(100), 327(44), 289(39), 287(50)$ & Proanthocyanidin dimer (A-type) & & $\checkmark$ & \\
\hline 78 & 29.0 & 477 & $\begin{array}{l}\mathrm{MS}^{2} \text { [477]: } 315(100), 314(91), 285(22), 271(16) \\
\mathrm{MS}^{3}[477 \rightarrow 315]: 301(96), 300(100), 285(82), 271(58)\end{array}$ & Isorhamnetin-O-hexoside & & $\checkmark$ & \\
\hline 81 & 30.2 & 447 & $\begin{array}{l}\mathrm{MS}^{2}[447]: 301(100) 1 \\
\operatorname{MS}^{3}[447 \rightarrow 301]: 271(24), 179(100), 151(94)\end{array}$ & Quercetin-O-deoxyhesoxide & $\checkmark$ & $\checkmark$ & \\
\hline 82 & 30.2 & 417 & $\begin{array}{l}\mathrm{MS}^{2} \text { [417]: } 327(22), 285(42), 284(100), 255(12), 227(7) \\
\left.\mathrm{MS}^{3} \text { [417 } \rightarrow 285\right]: 255(100), 229(12)\end{array}$ & Kaempferol-O-pentoside & $\checkmark$ & $\checkmark$ & \\
\hline 83 & 30.6 & 861 & $\begin{array}{l}\mathrm{MS}^{2} \text { [861]: } 735(100), 693(28), 575(61), 571(98), 539(45), 449 \\
(29), 447(18) \\
\mathrm{MS}^{3}[861 \rightarrow 735]: 717(46), 613(22), 575(70), 573(16), 553(50) \text {, } \\
487(37), 445(100), 409(33)\end{array}$ & (Epi)catechin-(epi)catechin-(epi)catechin (A-type) & & & $\checkmark$ \\
\hline 85 & 31.8 & 623 & $\begin{array}{l}\operatorname{MS}^{2}[623]: 315(100), 300(35) \\
\operatorname{MS}^{3}[623 \rightarrow 315]: 300(100), 271(8) \\
M^{4}[623 \rightarrow 315 \rightarrow 300]: 271(76), 255(100), 151(66)\end{array}$ & $\begin{array}{l}\text { Isorhamnetin- } O \text {-rutinoside or isorhamnetin- } O \text { - } \\
\text { neohesperidoside }\end{array}$ & $\checkmark$ & $\checkmark$ & \\
\hline 89 & 33.1 & 431 & $\begin{array}{l}\operatorname{MS}^{2}[431]: 285(100), 284(40), 255(5), 151(5) \\
\operatorname{MS}^{3}[431 \rightarrow 285]: 285(100), 257(50), 255(89), 241(15), 229(46) \\
151(11)\end{array}$ & Kaempferol-O-coumaroyl & $\checkmark$ & $\checkmark$ & $\checkmark$ \\
\hline 95 & 40.5 & 327 & $\mathrm{MS}^{2}$ [327]: 291 (53), 229 (78), 211 (30), 171 (100), 165 (26) & Oxo-dihydroxy-octadecenoic acid & $\checkmark$ & $\checkmark$ & $\checkmark$ \\
\hline 96 & 43.0 & 329 & MS $^{2}$ [329]: 311 (29), 293 (45), 229 (28), 211 (55), 171 (100) & Trihydroxyoctadecenoic acid & $\checkmark$ & $\checkmark$ & $\checkmark$ \\
\hline 97 & 45.7 & 723 & $\begin{array}{l}\text { MS }^{2} \text { [723]: } 577(59), 559(20), 437(76), 285(100) \\
\left.\text { MS }^{3} \text { [723 } \rightarrow 285\right]: 285(100), 257(13), 255(16), 241(38), 151(72)\end{array}$ & Kaempferol derivative & & $\checkmark$ & \\
\hline
\end{tabular}

loss of $132 \mathrm{Da}$ (pentoside), the aglycone ( $\mathrm{m} / \mathrm{z} 389)$ suffered several neutral losses of $15 \mathrm{Da}$ (methyl), so it was tentatively characterized as a polymethoxylated flavonoid- $O$-pentoside.

Four kaempferol derivatives were identified, based on the aglycone at $m / z$ 285. Compounds 72, 74, 84 and 89 suffered neutral losses of $308,162,162+162$, and $146 \mathrm{Da}$, respectively, and were characterized as kaempferol-O-rutinoside, kaempferol-O-hexoside, kaempferol-O-coumaroylhexoside, and kaempferol-O-coumaroyl, respectively. 
Table 3

Characterization of the methanol extract of leaves from A. barbujana (AB), O. foetens (OF) and P. indica (PI).

\begin{tabular}{|c|c|c|c|c|c|c|c|}
\hline No. & $\mathrm{t}_{R}(\min )$ & {$[\mathrm{M}-\mathrm{H}]^{-} m / z$} & $m / z$ (\% base peak) & Assigned identification & $\mathrm{AB}$ & $\mathrm{OF}$ & PI \\
\hline 2 & 3.4 & 487 & $\begin{array}{l}\mathrm{MS}^{2}[487]: 341(100) \\
\mathrm{MS}^{3}[487 \rightarrow 341]: 179(100), 161(16), 143(18), 131(7), 119(12) \\
113(14), 101(6) \\
\mathrm{MS}^{4}[487 \rightarrow 341 \rightarrow 179]: 161(36), 131(37), 119(58), 113(42) \\
101(36), 89(75)\end{array}$ & Saccharide & $\checkmark$ & $\checkmark$ & \\
\hline 3 & 3.5 & 683 & $\begin{array}{l}\mathrm{MS}^{2}[683]: 341(100) \\
\mathrm{MS}^{3}[683 \rightarrow 341]: 179(100), 161(20), 131(5), 119(35), 113(17) \\
\mathrm{MS}^{4}[683 \rightarrow 341 \rightarrow 179]: 161(100), 149(35), 143(26), 119(36) \\
113(21), 89(40)\end{array}$ & Saccharide & $\checkmark$ & & $\checkmark$ \\
\hline 4 & 3.6 & 533 & $\begin{array}{l}\operatorname{MS}^{2}[533]: 191(100), 173(2) \\
\operatorname{MS}^{3}[533 \rightarrow 191]: 173(14), 171(23), 127(100), 125(12), 93(68) \\
85(53) \\
M^{4}[533 \rightarrow 191 \rightarrow 127]: 109(100)\end{array}$ & Quinic acid derivative & $\checkmark$ & & $\checkmark$ \\
\hline 5 & 4.0 & 191 & $\operatorname{MS}^{2}$ [191]: 173 (54), 127 (100), 111 (76), 93 (35) & Quinic acid & $\checkmark$ & $\checkmark$ & $\checkmark$ \\
\hline 6 & 4.8 & 465 & $\begin{array}{l}\mathrm{MS}^{2}[465]: 375(9), 345(100) \\
\mathrm{MS}^{3}[465 \rightarrow 345]: 327(100), 317(25), 167(53) \\
\mathrm{MS}^{4}[465 \rightarrow 345 \rightarrow 327]: 309(30), 283(39), 151(100)\end{array}$ & Unknown & & & $\checkmark$ \\
\hline 9 & 6.3 & 465 & $\begin{array}{l}\mathrm{MS}^{2}[465]: 375(8), 345(100), 327(12) \\
\mathrm{MS}^{3}[465 \rightarrow 345]: 327(100), 317(21), 167(53), 151(8) \\
\mathrm{MS}^{4}[465 \rightarrow 345 \rightarrow 327]: 283(30), 201(19), 175(61), 165(28), \\
151(100)\end{array}$ & Unknown & & & $\checkmark$ \\
\hline 11 & 7.1 & 451 & $\begin{array}{l}\mathrm{MS}^{2}[451]: 417(15), 405(100) \\
\operatorname{MS}^{3}[451 \rightarrow 405]: 241(100), 224(60), 179(39), 161(9)\end{array}$ & Saccharide & & $\checkmark$ & \\
\hline 13 & 7.3 & 315 & $\begin{array}{l}\operatorname{MS}^{2}[315]: 153(100) \\
\operatorname{MS}^{3}[315 \rightarrow 153]: 109(100)\end{array}$ & Dihydroxybenzoic acid-O-hexoside & $\checkmark$ & & \\
\hline 14 & 7.7 & 353 & $\begin{array}{l}\mathrm{MS}^{2}[353]: 191(100), 179(36), 135(17) \\
\mathrm{MS}^{3}[353 \rightarrow 191]: 173(96), 127(100)\end{array}$ & 3-O-caffeoylquinic acid & $\checkmark$ & & \\
\hline 16 & 7.8 & 451 & $\begin{array}{l}\mathrm{MS}^{2}[451]: 405(100) \\
\mathrm{MS}^{3}[451 \rightarrow 405]: 179(100), 161(30), 149(8), 143(32), 131(13) \\
119(17)\end{array}$ & Saccharide (formate adduct) & $\checkmark$ & $\checkmark$ & \\
\hline 17 & 8.1 & 577 & $\mathrm{MS}^{2}$ [577]: 451 (32), 425 (100), 407 (89), 289 (25), 287 (11) & (Epi)catechin-(epi)catechin (B-type) & & & $\checkmark$ \\
\hline 18 & 8.8 & 449 & $\begin{array}{l}\mathrm{MS}^{2}[449]: 359(22), 329(100), 301(27) \\
\mathrm{MS}^{3}[449 \rightarrow 329]: 311(14), 301(100), 285(12), 167(13) \\
\mathrm{MS}^{4}[449 \rightarrow 329 \rightarrow 301]: 167(100)\end{array}$ & Unknown & & & $\checkmark$ \\
\hline 19 & 9.5 & 289 & $\mathrm{MS}^{2}$ [289]: $245(100), 205(48), 203(16), 179(21)$ & Catechin & & & $\checkmark$ \\
\hline 20 & 9.8 & 431 & $\begin{array}{l}\mathrm{MS}^{2}[431]: 391(17), 179(100), 161(15), 131(15) \\
\mathrm{MS}^{3}[431 \rightarrow 179]: 161(100), 149(57), 143(16), 113(35), 107(82) \\
89(44)\end{array}$ & Saccharide & & $\checkmark$ & \\
\hline 21 & 9.9 & 577 & $\mathrm{MS}^{2}$ [577]: 451 (20), 425 (100), 407 (70), 289 (25), 287 (12) & (Epi)catechin-(epi)catechin (B-type) & & $\checkmark$ & $\checkmark$ \\
\hline 22 & 10.1 & 865 & $\begin{array}{l}\mathrm{MS}^{2} \text { [865]: } 739(27), 713(30), 695(100), 543(25), 407(37), 287 \\
(24) \\
\left.\mathrm{MS}^{3} \text { [865 } \rightarrow 695\right]: 677(33), 543(100), 525(50), 451(53), 391(28) \\
289(14), 243(51) \\
\mathrm{MS}^{4}[865 \rightarrow 695 \rightarrow 543]: 525(100), 457(11), 373(10)\end{array}$ & (Epi)catechin-(epi)catechin-(epi)catechin (B-type) & & & $\checkmark$ \\
\hline 23 & 10.3 & 567 & $\begin{array}{l}\mathrm{MS}^{2}[567]: 522(28), 521(100) \\
\mathrm{MS}^{3}[567 \rightarrow 521]: 389(100), 293(12), 233(17), 227(12), 161(57) \\
\mathrm{MS}^{4}[567 \rightarrow 521 \rightarrow 389]: 227(8), 161(100), 143(7)\end{array}$ & Saccharide & $\checkmark$ & & \\
\hline 24 & 10.3 & 557 & $\begin{array}{l}\mathrm{MS}^{2}[557]: 522(21), 521(100) \\
\text { MS }^{3}[557 \rightarrow 521]: 389(100), 293(22), 179(10), 161(39) \\
\text { MS }^{4}[557 \rightarrow 521 \rightarrow 389]: 161(99), 131(73), 129(21), 115(100)\end{array}$ & Saccharide & $\checkmark$ & & \\
\hline 25 & 10.6 & 337 & $\begin{array}{l}\mathrm{MS}^{2}[337]: 163(100), 119(8) \\
\mathrm{MS}^{3}[337 \rightarrow 163]: 119(100)\end{array}$ & 3-p-coumaroylquinic acid & $\checkmark$ & & $\checkmark$ \\
\hline 26 & 10.6 & 435 & $\begin{array}{l}\mathrm{MS}^{2}[435]: 389(100), 345(29) \\
\mathrm{MS}^{3}[435 \rightarrow 389]: 273(14), 227(69), 161(100), 131(32) \\
\mathrm{MS}^{4}[435 \rightarrow 389 \rightarrow 161]: 143(100)\end{array}$ & Saccharide & $\checkmark$ & & \\
\hline 27 & 10.7 & 593 & $\begin{array}{l}\operatorname{MS}^{2}[593]: 503(43), 473(100), 383(36), 353(61) \\
\text { MS }^{3}[593 \rightarrow 473]: 353(100) \\
M^{4}[593 \rightarrow 473 \rightarrow 353]: 325(100), 297(31)\end{array}$ & Vicenin-2 (apigenin-6,8-di- $C$-glucoside) & & $\checkmark$ & \\
\hline 28 & 10.9 & 431 & $\begin{array}{l}\mathrm{MS}^{2}[431]: 225(31), 179(100), 161(14), 143(22) \\
\mathrm{MS}^{3}[431 \rightarrow 179]: 161(65), 143(99), 131(50), 119(64), 113(29) \\
89(100)\end{array}$ & Saccharide & $\checkmark$ & $\checkmark$ & $\checkmark$ \\
\hline 29 & 11.3 & 577 & $\begin{array}{l}\mathrm{MS}^{2} \text { [577]: } 559 \text { (12), } 451 \text { (22), } 425 \text { (92), } 407 \text { (100), } 289 \text { (21), } 287 \\
(12)\end{array}$ & (Epi)catechin-(epi)catechin (B-type) & $\checkmark$ & $\checkmark$ & $\checkmark$ \\
\hline 31 & 11.6 & 433 & $\begin{array}{l}\mathrm{MS}^{2}[433]: 387(100), 385(18) \\
\mathrm{MS}^{3}[433 \rightarrow 387]: 223(100), 205(72), 161(43), 153(71), 143(10) \\
\mathrm{MS}^{4}[433 \rightarrow 387 \rightarrow 223]: 205(44), 153(100)\end{array}$ & Dihydro-roseoside (formate adduct) & & & $\checkmark$ \\
\hline 32 & 11.9 & 431 & $\begin{array}{l}\mathrm{MS}^{2}[431]: 387(13), 385(100), 223(16) \\
\operatorname{MS}^{3}[431 \rightarrow 385]: 223(41), 205(78), 161(25), 153(100)\end{array}$ & Roseoside (formate adduct) & & & $\checkmark$ \\
\hline 34 & 12.6 & 863 & $\begin{array}{l}\mathrm{MS}^{2}[863]: 712(32), 711(100), 693(20), 559(27), 411(28) \\
\mathrm{MS}^{3}[863 \rightarrow 711]: 693(100), 559(77), 541(30), 407(13) \\
\mathrm{MS}^{4}[863 \rightarrow 711 \rightarrow 693]: 657(32), 567(100), 525(22), 407(31)\end{array}$ & (Epi)catechin-(epi)catechin-(epi)catechin (A-type) & & $\checkmark$ & $\checkmark$ \\
\hline 35 & 13.0 & 289 & $\mathrm{MS}^{2}$ [289]: 245 (100), 205 (39), 203 (19) & Epicatechin & $\checkmark$ & & $\checkmark$ \\
\hline 37 & 13.5 & 435 & $\mathrm{MS}^{2}$ [435]: $390(24), 389(100)$ & Saccharide & $\checkmark$ & & \\
\hline
\end{tabular}

$\mathrm{MS}^{3}$ [435 $\rightarrow$ 389]: $161(100), 159$ (10), 143 (6) 
Table 3 (continued)

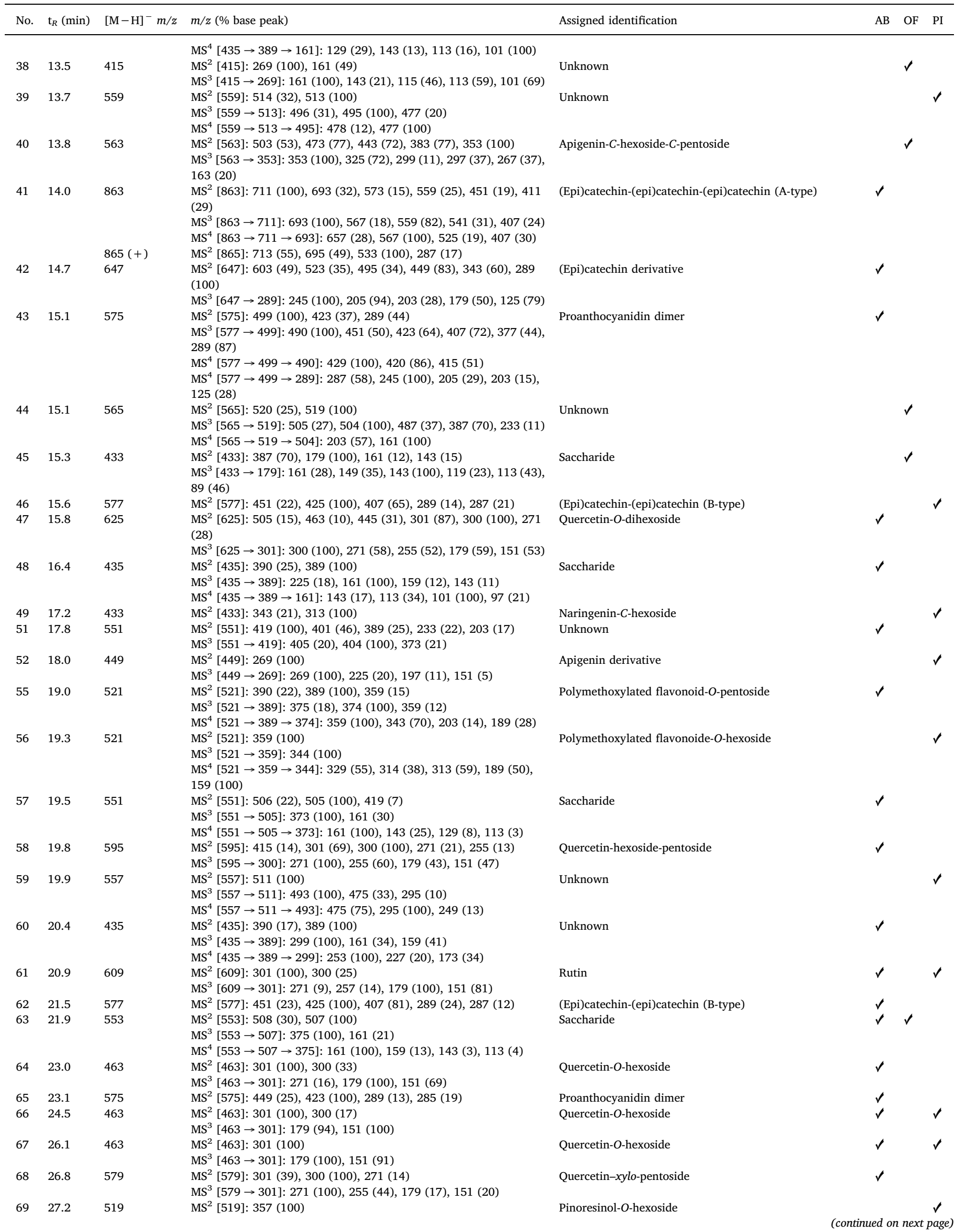


Table 3 (continued)

\begin{tabular}{|c|c|c|c|c|c|c|c|}
\hline No. & $\mathrm{t}_{R}(\min )$ & {$[\mathrm{M}-\mathrm{H}]^{-} m / z$} & $m / z$ (\% base peak) & Assigned identification & $\mathrm{AB}$ & $\mathrm{OF}$ & PI \\
\hline 70 & 27.5 & 473 & $\begin{array}{l}\mathrm{MS}^{3}[519 \rightarrow 357]: 342(8), 311(2), 151(100), 136(35) \\
\mathrm{MS}^{2}[473]: 428(27), 427(100), 293(28) \\
\mathrm{MS}^{3}[473 \rightarrow 427]: 293(100), 233(13), 125(41) \\
\mathrm{MS}^{4}[473 \rightarrow 427 \rightarrow 293]: 233(100), 161(10), 149(15), 131(6), \\
113(23), 99(28), 89(20)\end{array}$ & Saccharide & & $\checkmark$ & \\
\hline 71 & 27.7 & 609 & $\begin{array}{l}\mathrm{MS}^{2} \text { [609]: } 301(34), 300(100), 271(25) \\
\mathrm{MS}^{3}[609 \rightarrow 300]: 271(100), 255(79), 179(20), 151(21)\end{array}$ & Quercetin-O-neohesperidoside & $\checkmark$ & & \\
\hline 72 & 28.3 & 593 & $\begin{array}{l}\mathrm{MS}^{2}[593]: 285(100) \\
\mathrm{MS}^{3}[593 \rightarrow 285]: 257 \text { (100), } 229 \text { (99), } 107(66)\end{array}$ & Kaempferol-O-rutinoside & $\checkmark$ & & $\checkmark$ \\
\hline 73 & 28.5 & 433 & $\begin{array}{l}\mathrm{MS}^{2} \text { [433]: } 301(100), 300(75) \\
\left.\mathrm{MS}^{3} \text { [433 } \rightarrow 301\right]: 271(81), 255(62), 179(84), 151(100)\end{array}$ & Quercetin-O-pentoside & $\checkmark$ & & \\
\hline 74 & 28.7 & 447 & $\begin{array}{l}\mathrm{MS}^{2}[447]: 327(14), 285(92), 284(100), 255(22) \\
\mathrm{MS}^{3}[447 \rightarrow 284]: 257(30), 255(100), 213(12)\end{array}$ & Kaempferol-O-hexoside & $\checkmark$ & & $\checkmark$ \\
\hline 76 & 28.8 & 417 & $\begin{array}{l}\operatorname{MS}^{2}[417]: 371(100) \\
\operatorname{MS}^{3}[417 \rightarrow 371]: 209(10), 161(100), 113(15)\end{array}$ & Saccharide (formate adduct) & & & $\checkmark$ \\
\hline 77 & 28.8 & 503 & $\begin{array}{l}\mathrm{MS}^{2}[503]: 457(100), 293(42) \\
\mathrm{MS}^{3}[503 \rightarrow 457]: 293(100), 233(8) \\
\mathrm{MS}^{4}[503 \rightarrow 457 \rightarrow 293]: 233(65), 191(75), 149(84), 131(100) \\
89(50)\end{array}$ & Unknown & & $\checkmark$ & \\
\hline 79 & 29.6 & 551 & $\begin{array}{l}\mathrm{MS}^{2}[551]: 444(17), 443(100) \\
\mathrm{MS}^{3}[551 \rightarrow 443]: 153(100), 135(12) \\
\mathrm{MS}^{4}[551 \rightarrow 443 \rightarrow 153]: 109(100)\end{array}$ & Dihydroxybenzoic acid derivative & $\checkmark$ & & \\
\hline 80 & 30.1 & 543 & $\begin{array}{l}\mathrm{MS}^{2} \text { [543]: } 457(100), 383(19), 363(61), 345(42), 251(39), 237 \\
(44) \\
\mathrm{MS}^{3}[543 \rightarrow 457]: 413(100), 277(20), 233(25) \\
\mathrm{MS}^{4}[543 \rightarrow 457 \rightarrow 413]: 251(29), 233(100), 205(6), 189(12)\end{array}$ & Unknown & & & $\checkmark$ \\
\hline 81 & 30.2 & 447 & $\begin{array}{l}\mathrm{MS}^{2} \text { [447]: } 301(100), 300(18) \\
\mathrm{MS}^{3}[447 \rightarrow 301]: 271(11), 179(100), 151(98)\end{array}$ & Quercetin-O-deoxyhexoside & $\checkmark$ & & $\checkmark$ \\
\hline 83 & 30.6 & 861 & $\begin{array}{l}\left.\mathrm{MS}^{2}[861]: 825(15), 735100\right), 575(96), 571(51), 449(37) \\
\mathrm{MS}^{3}[861 \rightarrow 575]: 449(100), 423(15), 289(33)\end{array}$ & (Epi)catechin-(epi)catechin-(epi)catechin (A-type) & & $\checkmark$ & \\
\hline 84 & 31.3 & 593 & $\begin{array}{l}\mathrm{MS}^{2}[593]: 431(12), 413(66), 285(100), 255(30) \\
\operatorname{MS}^{3}[593 \rightarrow 285]: 257(30), 255(100), 229(9), 227(9), 151(7)\end{array}$ & Kaempferol-O-coumaroylhexoside & $\checkmark$ & & \\
\hline 85 & 31.8 & 623 & $\begin{array}{l}\mathrm{MS}^{2}[623]: 315(100), 300(87), 271(28) \\
\mathrm{MS}^{3}[623 \rightarrow 315]: 300(100) \\
\mathrm{MS}^{4}[623 \rightarrow 315 \rightarrow 300]: 271(100), 255(34), 151(5)\end{array}$ & $\begin{array}{l}\text { Isorhamnetin- } O \text {-rutinoside or isorhamnetin- } O \text { - } \\
\text { neohesperidoside }\end{array}$ & $\checkmark$ & & \\
\hline 86 & 32.4 & 475 & $\begin{array}{l}\operatorname{MS}^{2}[475]: 460(75), 313(100) \\
\operatorname{MS}^{3}[475 \rightarrow 313]: 298(100), 283(81) \\
M^{4}[475 \rightarrow 313 \rightarrow 298]: 283(100)\end{array}$ & Polymethoxylated flavonoid-O-hexoside & & $\checkmark$ & \\
\hline 87 & 32.9 & 375 & $\begin{array}{l}\mathrm{MS}^{2}[375]: 241(100) \\
\operatorname{MS}^{3}[375 \rightarrow 241]: 223(71), 151(23), 139(56), 97(100)\end{array}$ & Unknown & $\checkmark$ & & \\
\hline 88 & 33.1 & 441 & $\begin{array}{l}\mathrm{MS}^{2}[441]: 307(100), 295(23), 235(22), 159(22), 133(31) \\
\mathrm{MS}^{3}[441 \rightarrow 307]: 163(100)\end{array}$ & Unknown & & $\checkmark$ & \\
\hline 89 & 33.1 & 431 & $\begin{array}{l}\mathrm{MS}^{2}[431]: 285(100), 284(25) \\
\mathrm{MS}^{3}[431 \rightarrow 285]: 257(47), 255(100), 229(29)\end{array}$ & Kaempferol-O-coumaroyl & $\checkmark$ & & $\checkmark$ \\
\hline 90 & 33.8 & 461 & $\begin{array}{l}\operatorname{MS}^{2}[461]: 315(67), 314(100), 300(30), 299(84) \\
\operatorname{MS}^{3}[461 \rightarrow 315]: 300(61), 299(100) \\
M^{4}[461 \rightarrow 315 \rightarrow 299]: 271(100), 255(20), 151(10)\end{array}$ & Isorhamnetin-O-coumaroyl & $\checkmark$ & & \\
\hline 91 & 35.0 & 375 & $\begin{array}{l}\mathrm{MS}^{2}[375]: 241(100), 139(20) \\
\mathrm{MS}^{3}[375 \rightarrow 241]: 223(49), 151(15), 139(48), 97(100) \\
\mathrm{MS}^{4}[375 \rightarrow 241 \rightarrow 223]: 205(36), 193(77), 141(36), 125(68), \\
113(100)\end{array}$ & Unknown & & $\checkmark$ & \\
\hline 92 & 35.5 & 607 & $\begin{array}{l}\mathrm{MS}^{2}[607]: 299(100), 284(44), 255(22) \\
\mathrm{MS}^{3}[607 \rightarrow 299]: 284(100) \\
\mathrm{MS}^{4}[607 \rightarrow 299 \rightarrow 284]: 255(100), 227(6), 151(2)\end{array}$ & Diosmin & $\checkmark$ & & \\
\hline 93 & 38.4 & 445 & $\begin{array}{l}\mathrm{MS}^{2}[445]: 299(45), 298(100), 283(47) \\
\mathrm{MS}^{3}[445 \rightarrow 298]: 284(25), 283(100), 255(83) \\
\mathrm{MS}^{4}[445 \rightarrow 298 \rightarrow 283]: 255(100)\end{array}$ & Kaempferide-O-coumaroyl & $\checkmark$ & & \\
\hline 94 & 39.8 & 601 & $\begin{array}{l}\operatorname{MS}^{2}[601]: 555(100), 393(64) \\
\operatorname{MS}^{3}[601 \rightarrow 555]: 393(100) \\
M^{4}[601 \rightarrow 555 \rightarrow 393]: 209(18), 183(100), 139(10)\end{array}$ & Unknown & & $\checkmark$ & \\
\hline 95 & 40.5 & 327 & $\operatorname{MS}^{2}$ [327]: 291 (18), $229(11), 211(6), 171(100)$ & Oxo-dihydroxy-octadecenoic acid & $\checkmark$ & $\checkmark$ & $\checkmark$ \\
\hline 96 & 43.0 & 329 & $\begin{array}{l}\text { MS }^{2} \text { [329]: } 327 \text { (16), } 311 \text { (15), } 309 \text { (19), } 229 \text { (26), } 211 \text { (20), } 171 \\
(100)\end{array}$ & Trihydroxyoctadecenoic acid & $\checkmark$ & $\checkmark$ & $\checkmark$ \\
\hline
\end{tabular}

Compound 90 suffered a neutral losses of $146 \mathrm{Da}$; therefore it was characterized as isorhamnetin-O-coumaroyl.

Compound 93 exhibited the deprotonated molecular ion at $m / z 445$. It suffered the neutral loss of $146 \mathrm{Da}$ to yield the aglycone at $\mathrm{m} / z 299$. The aglycone was characterized as kaempferide (Engels et al., 2012). Considering the high retention time, it was assigned to kaempferide- $O$ coumaroyl.

3.1.3.3 O. foetens. Only three flavonoid glycosides were characterized in these extracts. Compound 27, with $[\mathrm{M}-\mathrm{H}]^{-}$at $\mathrm{m} / \mathrm{z}$ 593, was identified as vicenin-2 (Llorent-Martínez et al., 2015a). Compound 40 exhibited the deprotonated molecular ion at $m / z 563$, and presented an $\mathrm{MS}^{2}$ fragmentation pattern typical of asymmetrical di-C-glycosides, with fragment ions at $[\mathrm{M}-\mathrm{H}-210]^{-},[\mathrm{M}-\mathrm{H}-90]^{-}$and $[\mathrm{M}-\mathrm{H}-60]^{-}$; it was characterized as apigenin- $C$-pentoside- $C$ hexoside (Llorent-Martínez et al., 2015b). Finally, compound 86 presented $[\mathrm{M}-\mathrm{H}]^{-}$at $\mathrm{m} / z \quad 475$ and, after the loss of $162 \mathrm{Da}$, displayed the aglycone at $m / z 313$, which suffered several losses of 
<smiles>Oc1cc(O)c2c(c1)O[C@@]1(c3ccc(O)c(O)c3)Oc3cc(O)c4c(c3[C@@H]1O2)O[C@H](c1ccc(O)c(O)c1)[C@H](O)C4</smiles>

Procyanidin dimer (A type)<smiles>Oc1cc(O)c2c(c1)O[C@H](c1ccc(O)c(O)c1)[C@H](O)[C@H]2c1c(O)cc(O)c2c1O[C@H](c1ccc(O)c(O)c1)[C@H](O)C2</smiles>

Procyanidin dimer (B type)

Fig. 3. Procyanidin dimers: A-type and B-type.

Table 4

Quantification of phenolic compounds in leaves of the analyzed plant species ( $\left.\mathrm{mg} \mathrm{g}^{-1} \mathrm{DE}\right)$.

\begin{tabular}{|c|c|c|c|c|c|c|c|}
\hline \multirow[t]{2}{*}{$\mathrm{N}^{\circ}$} & \multirow[t]{2}{*}{ Assigned identification } & \multicolumn{3}{|l|}{ L. novocanariensis } & \multirow[t]{2}{*}{ A. barbujana } & \multirow[t]{2}{*}{ O. foentes } & \multirow[t]{2}{*}{ P. indica } \\
\hline & & L1 & $\mathrm{L} 2$ & L3 & & & \\
\hline \multicolumn{8}{|c|}{ Phenolic acids } \\
\hline 12 & Dihydroxybenzoic acid- $O$-hexoside & $0.52 \pm 0.02$ & $0.56 \pm 0.04$ & & & & \\
\hline 25 & 3-p-Coumaroylquinic acid & & & & & & $5.60 \pm 0.09$ \\
\hline Total & & $0.52 \pm 0.02^{\mathrm{a}}$ & $0.56 \pm 0.04^{\mathrm{a}}$ & & & & $5.60 \pm 0.09^{b}$ \\
\hline \multicolumn{8}{|l|}{ Flavonols } \\
\hline 47 & Quercetin-O-dihexoside & & & & $0.52 \pm 0.13$ & & \\
\hline 58 & Quercetin-hexoside-pentoside & & & & $0.45 \pm 0.03$ & & \\
\hline 61 & Rutin & & & & & & $0.76 \pm 0.01$ \\
\hline 64 & Quercetin- $O$-hexoside & & & & $1.83 \pm 0.01$ & & \\
\hline 66 & Quercetin- $O$-hexoside & & & & $1.97 \pm 0.07^{\mathrm{b}}$ & & $0.40 \pm 0.01^{\mathrm{a}}$ \\
\hline 67 & Quercetin-O-hexoside & & & & $1.81 \pm 0.01^{\mathrm{b}}$ & & $0.75 \pm 0.01^{\mathrm{a}}$ \\
\hline 73 & Quercetin- $O$-pentoside & & & & $1.82 \pm 0.12$ & & \\
\hline 74 & Kaempferol-O-hexoside & $1.19 \pm 0.10$ & & & & & \\
\hline 81 & Quercetin-O-deoxyhesoxide & $1.50 \pm 0.06^{\mathrm{b}}$ & $0.87 \pm 0.01^{\mathrm{a}}$ & & $3.69 \pm 0.21^{\mathrm{d}}$ & & $2.14 \pm 0.01^{\mathrm{c}}$ \\
\hline 82 & Kaempferol-O-pentoside & & $0.40 \pm 0.02$ & & & & \\
\hline 84 & Kaempferol-O-coumaroylhexoside & & & & $0.61 \pm 0.03$ & & \\
\hline 85 & Isorhamnetin- $O$-rutinoside & & & & $0.30 \pm 0.01$ & & \\
\hline 89 & Kaempferol-O-coumaroyl & & $0.83 \pm 0.04^{\mathrm{a}}$ & & $1.27 \pm 0.01^{\mathrm{b}}$ & & $3.31 \pm 0.04^{\mathrm{c}}$ \\
\hline 97 & Kaempferol derivative & & $10.33 \pm 0.50$ & & & & \\
\hline Total & & $2.69 \pm 0.16^{\mathrm{a}}$ & $12.43 \pm 0.58^{\mathrm{c}}$ & & $14.29 \pm 0.62^{d}$ & & $7.36 \pm 0.01^{\mathrm{b}}$ \\
\hline \multicolumn{8}{|l|}{ Flavanols } \\
\hline 15 & Proanthocyanidin dimer & $4.31 \pm 0.21^{\mathrm{b}}$ & $4.43 \pm 0.21^{\mathrm{b}}$ & $0.62 \pm 0.01^{\mathrm{a}}$ & & & \\
\hline 17 & (Epi)catechin-(epi)catechin (B-type) & & & & & & $8.91 \pm 0.38$ \\
\hline 21 & (Epi)catechin-(epi)catechin (B-type) & $4.04 \pm 0.05^{\mathrm{c}}$ & $3.91 \pm 0.10^{\mathrm{c}}$ & $1.23 \pm 0.07^{\mathrm{b}}$ & & & $0.76 \pm 0.04^{\mathrm{a}}$ \\
\hline 29 & (Epi)catechin-(epi)catechin (B-type) & & & & $4.66 \pm 0.06$ & & \\
\hline 34 & A-type proanthocyanidin trimer & $48.80 \pm 0.85^{\mathrm{d}}$ & $34.13 \pm 1.38^{\mathrm{b}}$ & $40.11 \pm 0.30^{c}$ & & $17.45 \pm 0.47^{\mathrm{a}}$ & \\
\hline 35 & Epicatechin & $5.23 \pm 0.49^{\mathrm{b}}$ & $7.00 \pm 0.34^{c}$ & $0.10 \pm 0.06^{\mathrm{a}}$ & $5.20 \pm 0.18^{b}$ & & \\
\hline 36 & Proanthocyanidin dimer & & $1.08 \pm 0.06$ & & & & \\
\hline 41 & (Epi)catechin-(epi)catechin-(epi)catechin (A-type) & & & & $62.66 \pm 1.81$ & & \\
\hline 43 & Proanthocyanidin dimer & & & & $1.56 \pm 0.05$ & & \\
\hline 65 & Proanthocyanidin dimer & $2.21 \pm 0.09^{c}$ & $1.25 \pm 0.01^{\mathrm{a}}$ & $2.02 \pm 0.09^{b}$ & & & \\
\hline 83 & (Epi)catechin-(epi)catechin-(epi)catechin (A-type) & & & $3.45 \pm 0.01^{b}$ & & $0.90 \pm 0.05^{\mathrm{a}}$ & \\
\hline 89 & Kaempferol-O-coumaroyl & & $0.83 \pm 0.04^{\mathrm{a}}$ & & $1.27 \pm 0.01^{\mathrm{b}}$ & & $3.31 \pm 0.04^{\mathrm{c}}$ \\
\hline Total & & $64.59 \pm 1.70^{\mathrm{d}}$ & $51.80 \pm 2.01^{\mathrm{c}}$ & $48.45 \pm 0.54^{\mathrm{c}}$ & $74.09 \pm 2.10^{\mathrm{e}}$ & $18.35 \pm 0.52^{b}$ & $9.67 \pm 0.33^{\mathrm{a}}$ \\
\hline \multicolumn{8}{|l|}{ Flavones } \\
\hline 27 & Vicenin-2 & & & & & $0.09 \pm 0.01$ & \\
\hline 50 & Rhamnosylvitexin & & $0.13 \pm 0.01$ & & & & \\
\hline 52 & Apigenin derivative & & & & & & $0.15 \pm 0.01$ \\
\hline 57 & 2"-O-rhamnosylvitexin & $0.23 \pm 0.02^{\mathrm{a}}$ & $0.48 \pm 0.01^{\mathrm{b}}$ & $0.22 \pm 0.01^{\mathrm{a}}$ & & & \\
\hline 92 & Diosmin & & & & $0.60 \pm 0.04$ & & \\
\hline Total & & $0.23 \pm 0.02^{b}$ & $0.58 \pm 0.02^{c}$ & $0.22 \pm 0.01^{\mathrm{b}}$ & $0.60 \pm 0.04^{\mathrm{c}}$ & $0.09 \pm 0.01^{\mathrm{a}}$ & $0.15 \pm 0.01^{\mathrm{a}}$ \\
\hline \multicolumn{8}{|l|}{ Flavanone } \\
\hline 49 & Naringenin- $C$-hexoside & & & & & & $2.28 \pm 0.06$ \\
\hline Total & & & & & & & $2.28 \pm 0.06$ \\
\hline TIPC & & $68.02 \pm 1.90^{\mathrm{d}}$ & $65.37 \pm 2.73^{d}$ & $48.67 \pm 0.55^{c}$ & $88.99 \pm 2.76^{\mathrm{e}}$ & $18.44 \pm 0.52^{\mathrm{a}}$ & $25.06 \pm 0.57^{b}$ \\
\hline Yield (\%) & & 4.38 & 7.96 & 4.53 & 4.62 & 4.48 & 9.84 \\
\hline
\end{tabular}

Means in the same line not sharing the same letter are significantly different at $p<0.05$ probability level. 
$15 \mathrm{Da}$, so it was tentatively characterized as the hexoside of a polymethoxylated flavonoid.

3.1.3.4 P. indica. Compounds $\mathbf{3 1}$ and $\mathbf{3 2}$ were characterized as the formic adducts of dihydro-roseoside and roseoside (drovomifoliol-Oglucoside), respectively, based on bibliographic information (Spínola and Castilho, 2016).

Compound 49, with $[\mathrm{M}-\mathrm{H}]^{-}$at $m / z$ 433, and fragment ions at $[\mathrm{M}-\mathrm{H}-90]^{-}$and $[\mathrm{M}-\mathrm{H}-120]^{-}$, was tentatively characterized as naringenin-C-hexoside (Vallverdú-Queralt et al., 2011).

Compound 52 exhibited $\mathrm{MS}^{3}$ fragment ions at $\mathrm{m} / \mathrm{z} 269$ and 225, typical of apigenin, so it was tentatively characterized as a derivative.

Compounds 66, 67 and 81 were quercetin glycosides (aglycone at $\mathrm{m} / \mathrm{z} 301$ ). 66 and 67 were quercetin-O-hexoside isomers, whereas 81 corresponded to quercetin- $O$-deoxyhexoside.

Compound 69 displayed an $[\mathrm{M}-\mathrm{H}]^{-}$ion at $m / z$ 519. It suffered the neutral loss of $162 \mathrm{Da}$. The aglycone, at $m / z 357$, presented fragment ions at $m / z 342,311,151$ and 136, typical from pinoresinol, so it was characterized as its $O$-hexoside (Ye et al., 2005).

\subsubsection{Other compounds}

A high number of compounds (Tables 2 and 3) presented $M^{\mathrm{n}}$ fragment ions at $m / z 131,119,113$ and 89, typical from saccharides (Brudzynski and Miotto, 2011; Verardo et al., 2009), so they were tentatively characterized as saccharide derivatives. These compounds were observed in all the analyzed plants.

Compound 5, $[\mathrm{M}-\mathrm{H}]^{-}$at $m / z 191$, exhibited $\mathrm{MS}^{2}$ fragment ions at $\mathrm{m} / z 173$ and 111, typical from quinic acid (Llorent-Martínez et al., 2015a).

Compound 7 displayed $[\mathrm{M}-\mathrm{H}]^{-}$at $m / z 315$ and two fragment ions at $m / z 153$ and 123, consistent with hydroxytyrosol hexoside (Savarese et al., 2007)

Compound 12 exhibited $[\mathrm{M}-\mathrm{H}]^{-}$at $m / z 315$, and $\mathrm{MS}^{\mathrm{n}}$ fragment ions at $m / z 153$ and 109, which has been previously mentioned as typical from a dihydroxybenzoic acid-O-hexoside (Han et al., 2008).

Compound 13, detected in A. barbujana, exhibited $[\mathrm{M}-\mathrm{H}]^{-}$at $m / z$ 315 , and $M S^{\mathrm{n}}$ fragment ions at $m / z 153$ and 109, previously mentioned as typical of dihydroxybenzoic acid-O-hexoside (Han et al., 2008).

Compound 25, in $P$. indica, exhibited the deprotonated molecular ion at $m / z 337$, and $\mathrm{MS}^{2}$ base peak ion at $m / z 163$, so it was characterized as 3-p-coumaroylquinic acid (Clifford et al., 2003).

Compounds 95 and 96, detected in all plants, were tentatively characterized as oxo-dihydroxy-octadecenoic and trihydroxy-octadecenoic acids, respectively, considering bibliographic data (Van Hoyweghen et al., 2014).

\subsection{Quantification of individual polyphenols}

In the present study, 34 polyphenols were quantified by HPLC-DAD (Table 4) using the corresponding standards for calibration for each group. Only the most abundant compounds were identified, since the overlapping of some compounds or their low levels did not allow to carry out an accurate quantification.

The most relevant arboreal species in Laurisilva forest are $L$. novocanariensis, $O$. foetens, A. barbujana and $P$. indica from the Lauraceae family, which gives name to the forest, and Olea europaea, Ilex perado, Clethra arborea, Heberdenia excelsa, Juniperus cedrus, Ilex canariensis and Myrica faya, from other families. In previous works, our research group characterized the phenolic fractions of several of these non-lauraceae species (Llorent-Martínez et al., 2015a; Spínola et al., 2014). Hence, comparison between several species of the Laurisilva forest will be performed.

In this work, intra and inter-species differences were observed for TIPC $(p<0.05)$. A. barbujana presented the higher phenolic contents and $O$. foetens the lowest ( 88.99 and $18.43 \mathrm{mg} \mathrm{g}^{-1} \mathrm{DE}$, respectively) (Table 4). This trend is in agreement with a previous study (Tavares et al., 2010), but no individual quantitative data was shown before. Significant differences $(p<0.05)$ were found in TIPC of Laurus collected in different locations of Madeira Island (48.67-68.02 $\mathrm{mg} \mathrm{g}^{-1} \mathrm{DE}$ ). Additionally, variations were also observed between Laurus samples for HCAs, flavonols, flavanols and flavones contents (Table 4). The lower phenolic amounts found in Ponta do Pargo samples (L3) may be related to different soil and climatic conditions, since these plants grown in the southside of the Island.

In non-lauraceae species, O. europaea profile was dominated by secoiridoids, mainly oleuropein derivatives; I. perado showed HCA esters and saponins as main components; $H$. excelsa was rich in flavonols and isoflavonols; and C. arborea contained several B-type procyanidin dimers and trimers, and flavonol derivatives as well (Llorent-Martínez et al., 2015a). In addition, M. faya presented mainly flavanols and flavonols (Spínola et al., 2014). Quantitative data in the present work indicated that flavanols (79.24-99.55\%) were the most representative class of polyphenols on Laurus samples, followed by flavonols (0-19.01\%), flavones and HCAs. Flavonoids were dominant in A. barbujana: flavanols $(83.26 \%)>$ flavonols $(16.05 \%)>$ flavones (0.68\%). Flavanols $(99.51 \%)$ and flavones $(0.48 \%)$ composed the polyphenolic profile of $O$. foetens. $P$. indica showed the most diverse phytochemical composition among analyzed plants: flavanols $(38.58 \%)>$ flavonols $(29.38 \%)>$ HCAs $(22.34 \%)>$ flavanones $(2.28 \%)>$ flavones $(0.58 \%)$.

A study conducted on P. americana leaves (Uysal et al., 2016) showed that chlorogenic acid was the main component (15.78-18.93 $\mathrm{mg} \mathrm{g}^{-1} \mathrm{DE}$ ), which was absent in the analyzed $P$. indica samples. Regarding other analyzed Lauraceae species, no data in literature were found about their phenolic compositions. M. faya leaves yielded a total of $178 \mathrm{mg} / 100 \mathrm{~g}$ dry weight (DW) of flavanols, mainly gallocatechin derivatives, but the main components were flavonols (1204 mg/100 g DW) (Spínola et al., 2014).

The most important discovery in all the analyzed extracts was the presence of PAs. In fact, PAs represented at least $80 \%$ of the phenolic profile in L. novocanariensis and A. barbujana. An A-type PA trimer (compound 34) was the most abundant phenolic in $L$. novocanariensis and $O$. foetens (17.45-48.80 $\left.\mathrm{mg} \mathrm{g}^{-1} \mathrm{DE}\right)$. (Epi)catechin-(epi)catechin(epi)catechin (A-type) (compound 41) was major in A. barbujana and (epi)catechin-(epi)catechin (B-type) (compound 17) in $P$. indica (62.66 and $8.91 \mathrm{mg} \mathrm{g}^{-1} \mathrm{DE}$, respectively).

Similarly to the present work, A-type PAs were also found as major phenolics in three Laurus species (including L. novocanariensis) (Vinha et al., 2015). By contrast, the same authors reported that PA dimers were more abundant than trimers. In general, the analyzed Laurus samples presented superior individual and total phenolic amounts than those studied by Vinha and co-workers (7.58-33.24 $\mathrm{mg} \mathrm{g}^{-1} \mathrm{DE}$ ) (Vinha et al., 2015). These differences may be related to differences in the collection area, sample preparation, time of the year, and even the sexual dimorphism of Laurus.

PAs are compounds of high interest due to their anti-infectious, antiinflammatory, cardioprotective and anticarcinogenic properties proved in clinical and laboratory studies (Nandakumar et al., 2008). These properties depend on the distribution of oligomers and polymers and on the type of proanthocyanidin: A or B depending on the number of bonds between catechin units and two and one linkage, respectively (Tsao, 2010).

PAs are found in plant material in a wide range of amounts (Kardel et al., 2013; Ropiak et al., 2016). However, only a few - such as plums, avocados, peanuts, cinnamon, and cranberries - contain A-type procyanidins. The major sources are some berries (blueberries, cranberries, and black currant) and plums (prunes), with a content of about $200 \mathrm{mg} / 100 \mathrm{~g}$ (fresh weight); cinnamon bark, which can reach $>2000$ mg/100 g (dry weight) (Gu et al., 2004) and maritime pine (Pinus pinaster) bark (Maimoona et al., 2011). Cinnamon (another Lauraceae) bark has been widely recognized for its different health benefits, mainly associated to its PAs (A-type) content (Mateos-Martín et al., 2012; Peng 

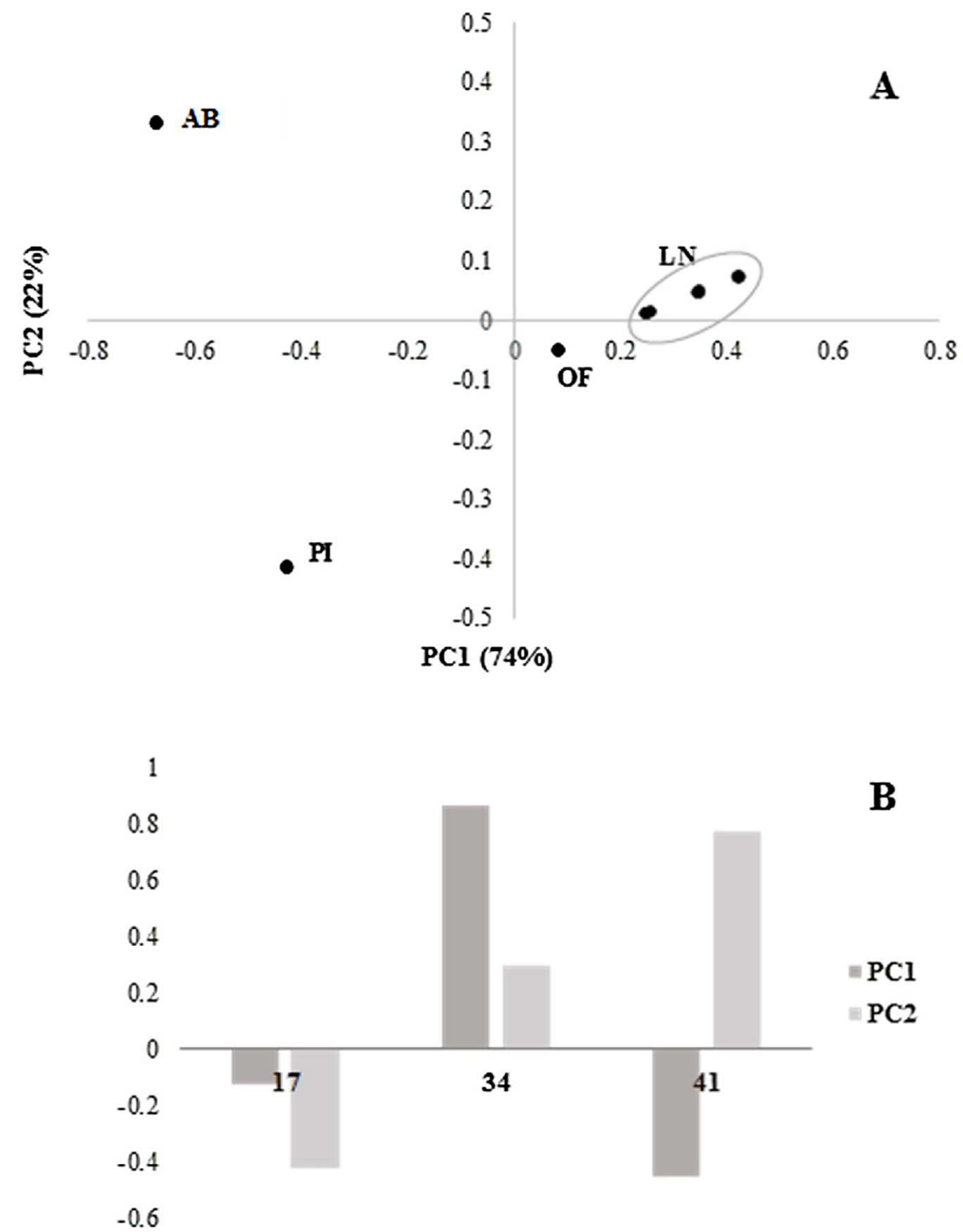

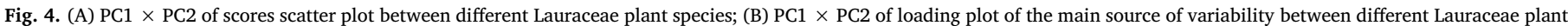
species. AB: A. barbujana; PI: P. indica; LN: L. novocanarienses; OF: O. Foetens.

et al., 2010; Rao and Gan, 2014; Sun et al., 2016), although the coumarin contained in cinnamon is known to be hepatotoxic (Iwata et al., 2016), hence the importance of finding new sources of PAs.

The A-type variety is more active tan B-type in reduction of cell proliferation, increase of apoptosis, cell cycle arrest and modulation of the expression and activity of NF-kB and NF-kB target genes. For instance, A-type procyanidins exhibit, in vitro, a capacity of inhibition of P-fimbriated Escherichia coli adhesion to uroepithelial cells greater than B-type procyanidins. In fact, different extracts containing only B-type procyanidins had no anti-adhesion activity (Howell et al., 2005).

\subsection{Principal component analysis}

PCA statistical tool was applied to the concentrations of 34 polyphenols determined by HPLC-DAD to establish a relationship between targeted plant species. The PCA score scatter plot of the two first principal components (which explains $96 \%$ of the total variability) is shown in Fig. 4.

The loadings of each compound (variable) that contribute to explain the differentiation between plant species is shown in Fig. 4B. PC1, that explained $74 \%$ of the total variability, shows Lauraceae species discrimination based on phenolic profile, where L. novocanariensis
(LN) and $O$. foetens (OF) samples are projected in PC1 positive, A. barbujana (AB) is above the positive PC2 axis and P. indica (PI) in PC2 negative. Taking into account the loading plot (Fig. 4B), the compounds responsible for these results were proanthocyanidins: (Epi)catechin(epi)catechin (B-type) (17), A-type proanthocyanidin trimer (34) and (Epi)catechin-(epi)catechin-(epi)catechin (A-type) (41).

\section{Conclusions}

A report on the phenolic composition of leaves of the most important Lauraceae plants of the Laurisilva forest, part of UNESCO patrimony, is presented; the most important compounds detected in the analyzed extracts were characterized and quantified.

It was observed that flavonoids, glycosides and proanthocyanidins (A- and B- type), were the most abundant compounds. In particular, the highest amounts of these compounds were observed in $L$. novocanariensis and A. barbujana, which present amounts of A-type PA trimers similar to those found in commercial sources. It has been reported that PA trimers are mainly responsible for the activity of cinnamon extracts (Sun et al., 2016). Therefore, an interesting direction for upcoming research will be the isolation and NMR identification of these trimers, and the study of their individual biological properties as well. Con- 
sidering the high number of health benefits that the identified compounds present, these species could be considered as potential novel sources of these compounds in the pharmaceutical industry. Hence, further research regarding antioxidant and toxicity assays will be performed in our group to improve the knowledge of these species.

\section{Acknowledgments}

E. J. Llorent-Martínez acknowledges the financial support from the UCLM Research Plan. V. Spínola is grateful to Fundação para a Ciência e a Tecnologia (FCT, Portugal) for a Ph.D. grant SFRH/BD/84672/ 2012. This research was supported by FCT with funds from the Portuguese Government (Project PEst-OE/QUI/UI0674/2013) and the Portuguese National Mass Spectrometry Network (Contract RNEMREDE/1508/REM/2005). Funding through the project M142001-0145-FEDER-000005 - Centro de Química da Madeira - CQM + (Madeira 14-20) is also acknowledged.

\section{References}

Abad-García, B., Garmón-Lobato, S., Berrueta, L.A., Gallo, B., Vicente, F., 2009. Practical guidelines for characterization of $O$-diglycosyl flavonoid isomers by triple quadrupole MS and their applications for identification of some fruit juices flavonoids. J. Mass Spectrom. 44, 1017-1025.

Alvárez, J.M., Cuca, L.E., Carrasco-Pancorbo, A., Ruiz-Muelle, A.B., Fernández, I., Fernández-Gutiérrez, A., 2016. Phenolic constituents of leaves from Persea caerulea Ruiz \& Pav; Mez (Lauraceae). Biochem. Syst. Ecol. 67, 53-57.

Appeldoorn, M.M., Vincken, J.-P., Sanders, M., Hollman, P.C.H., Gruppen, H., 2009. Combined normal-phase and reversed-phase liquid chromatography/ESI-MS as a tool to determine the molecular diversity of A-type procyanidins in peanut skins. J. Agric. Food Chem. 57, 6007-6013.

Bakr, R.O., El Bishbishy, M.H., 2016. Profile of bioactive compounds of Capparis spinosa var. aegyptiaca growing in Egypt. Rev. Bras. Farmacogn. 26, 514-520.

Brudzynski, K., Miotto, D., 2011. Honey melanoidins: analysis of the compositions of the high molecular weight melanoidins exhibiting radical-scavenging activity. Food Chem. 127, 1023-1030.

Clifford, M.N., Johnston, K.L., Knight, S., Kuhnert, N., 2003. Hierarchical scheme for LCMSn identification of chlorogenic acids. J. Agric. Food Chem. 51, 2900-2911.

Dou, J., Lee, V.S.Y., Tzen, J.T.C., Lee, M.-R., 2007. Identification and comparison of phenolic compounds in the preparation of oolong tea manufactured by semifermentation and drying processes. J. Agric. Food Chem. 55, 7462-7468.

Engels, C., Gräter, D., Esquivel, P., Jiménez, V.M., Gänzle, M.G., Schieber, A., 2012. Characterization of phenolic compounds in jocote (Spondias purpurea L.) peels by ultra high-performance liquid chromatography/electrospray ionization mass spectrometry. Food Res. Int. 46, 557-562.

Ferreres, F., Gil-Izquierdo, A., Andrade, P.B., Valentão, P., Tomás-Barberán, F.A., 2007 Characterization of $\mathrm{C}$-glycosyl flavones $\mathrm{O}$-glycosylated by liquid chromatography-tandem mass spectrometry. J. Chromatogr. A 1161, 214-223.

Gouveia, S.C., Castilho, P.C., 2013. Artemisia annua L.: essential oil and acetone extract composition and antioxidant capacity. Ind. Crops Prod. 45, 170-181.

Gruz, J., Novák, O., Strnad, M., 2008. Rapid analysis of phenolic acids in beverages by UPLC-MS/MS. Food Chem. 111, 789-794.

Gu, L., Kelm, M.A., Hammerstone, J.F., Beecher, G., Holden, J., Haytowitz, D., Gebhardt, S., Prior, R.L., 2004. Concentrations of proanthocyanidins in common foods and estimations of normal consumption. J. Nutr. 134, 613-617.

Han, J., Ye, M., Qiao, X., Xu, M., Wang, B.-R., Guo, D.-A., 2008. Characterization of phenolic compounds in the Chinese herbal drug Artemisia annua by liquid chromatography coupled to electrospray ionization mass spectrometry. J. Pharm. Biomed. Anal. 47, 516-525.

Howell, A.B., Reed, J.D., Krueger, C.G., Winterbottom, R., Cunningham, D.G., Leahy, M., 2005. A-type cranberry proanthocyanidins and uropathogenic bacterial anti-adhesion activity. Phytochemistry 66, 2281-2291.

Iwata, N., Kainuma, M., Kobayashi, D., Kubota, T., Sugawara, N., Uchida, A., Ozono, S., Yamamuro, Y., Furusyo, N., Ueda, K., Tahara, E., Shimazoe, T., 2016. The relation between hepatotoxicity and the total coumarin intake from traditional Japanese medicines containing cinnamon bark. Front. Pharmacol. 7, 174

Kajdžanoska, M., Gjamovski, V., Stefova, M., 2010. HPLC-DAD-ESI-MSn identification of phenolic compounds in cultivated strawberries from Macedonia. Maced. J. Chem. Chem. Eng. 29, 181-194.

Kardel, M., Taube, F., Schulz, H., Schütze, W., Gierus, M., 2013. Different approaches to evaluate tannin content and structure of selected plant extracts - review and new aspects. J. Appl. Bot. Food Qual. 86, 154-166.

Llorent-Martínez, E.J., Gouveia, S., Castilho, P.C., 2015a. Analysis of phenolic compounds in leaves from endemic trees from Madeira Island: a contribution to the chemotaxonomy of Laurisilva forest species. Ind. Crops Prod. 64, 135-151.

Llorent-Martínez, E.J., Spínola, V., Gouveia, S., Castilho, P.C., 2015b. HPLC-ESI-MSn characterization of phenolic compounds, terpenoid saponins, and other minor compounds in Bituminaria bituminosa. Ind. Crops Prod. 69, 80-90.
Määttä-Riihinen, Kaisu R, Kähkönen, Marja P., Törrönen, A. Riitta, Heinonen, I.M., 2005. Catechins and procyanidins in berries of Vaccinium species and their antioxidant activity. J. Agric. Food Chem. 53, 8485-8491.

Maimoona, A., Naeem, I., Saddiqe, Z., Jameel, K., 2011. A review on biological, nutraceutical and clinical aspects of French maritime pine bark extract. J. Ethnopharmacol. 133, 261-277.

Mateos-Martín, M.L., Fuguet, E., Quero, C., Pérez-Jiménez, J., Torres, J.L., 2012. New identification of proanthocyanidins in cinnamon (Cinnamomum zeylanicum L.) using MALDI-TOF/TOF mass spectrometry. Anal. Bioanal. Chem. 402, 1327-1336.

Nandakumar, V., Singh, T., Katiyar, S.K., 2008. Multi-targeted prevention and therapy of cancer by proanthocyanidins. Cancer Lett. 269, 378-387.

Peng, X., Ma, J., Chao, J., Sun, Z., Chang, R.C.-C., Tse, I., Li, E.T.S., Chen, F., Wang, M., 2010. Beneficial effects of cinnamon proanthocyanidins on the formation of specific advanced glycation endproducts and methylglyoxal-induced impairment on glucose consumption. J. Agric. Food Chem. 58, 6692-6696.

Press, J.R., Short, M.J., 1994. Flora of Madeira. HMSO, London.

Rao, P.V., Gan, S.H., 2014. Cinnamon: a multifaceted medicinal plant. Evid. Based Complement. Altern. Med. 2014, 642942.

Rivera, D., Obón, C., 1995. The ethnopharmacology of Madeira and Porto Santo Islands, a review. J. Ethnopharmacol. 46, 73-93.

Ropiak, H.M., Ramsay, A., Mueller-Harvey, I., 2016. Condensed tannins in extracts from European medicinal plants and herbal products. J. Pharm. Biomed. Anal. 121, 225-231.

Ruiz, D., Egea, J., Gil, M.I., Tomás-Barberán, F.A., 2005. Characterization and quantitation of phenolic compounds in new apricot (Prunus armeniaca L.) varieties. J. Agric. Food Chem. 53, 9544-9552.

Sánchez-Rabaneda, F., Jáuregui, O., Lamuela-Raventós, R.M., Viladomat, F., Bastida, J., Codina, C., 2004. Qualitative analysis of phenolic compounds in apple pomace using liquid chromatography coupled to mass spectrometry in tandem mode. Rapid Commun. Mass Spectrom. 18, 553-563.

Savarese, M., De Marco, E., Sacchi, R., 2007. Characterization of phenolic extracts from olives (Olea europaea cv. Pisciottana) by electrospray ionization mass spectrometry. Food Chem. 105, 761-770.

Soong, Y.Y., Barlow, P.J., 2005. Isolation and structure elucidation of phenolic compounds from longan (Dimocarpus longan Lour.) seed by high-performance liquid chromatography-electrospray ionization mass spectrometry. J. Chromatogr. A 1085, $270-277$.

Spínola, V., Castilho, P.C., 2016. Phytochemical profile, chemotaxonomic studies, and in vitro antioxidant activities of two endemisms from Madeira Archipelago: Melanoselinum decipiens and Monizia edulis (Apiaceae). Chem. Biodivers. 13 $1290-1306$.

Spínola, V., Llorent-Martínez, E.J., Gouveia, S., Castilho, P.C., 2014. Myrica faya: a new source of antioxidant phytochemicals. J. Agric. Food Chem. 62, 9722-9735.

Stöggl, W.M., Huck, C.W., Bonn, G.K., 2004. Structural elucidation of catechin and epicatechin in sorrel leaf extracts using liquid-chromatography coupled to diode array-, fluorescence-, and mass spectrometric detection. J. Sep. Sci. 27, 524-528.

Sun, P., Wang, T., Chen, L., Yu, B., Jia, Q., Chen, K., Fan, H., Li, Y., Wang, H., 2016. Trimer procyanidin oligomers contribute to the protective effects of cinnamon extracts on pancreatic $\beta$-cells in vitro. Acta Pharmacol. Sin. 37, 1083-1090.

Tavares, L., Carrilho, D., Tyagi, M., Barata, D., Serra, A.T., Duarte, C.M.M., Duarte, R.O., Feliciano, R.P., Bronze, M.R., Chicau, P., Espírito-Santo, M.D., Ferreira, R.B., dos Santos, C.N., 2010. Antioxidant capacity of Macaronesian traditional medicinal plants. Molecules 15, 2576-2592.

Tomás-Barberán, F.A., Gil, M.I., Cremin, P., Waterhouse, A.L., Hess-Pierce, B., Kader, A.A., 2001. HPLC-DAD-ESIMS analysis of phenolic compounds in nectarines, peaches, and plums. J. Agric. Food Chem. 49, 4748-4760.

Tsao, Rong, 2010. Chemistry and biochemistry of dietary polyphenols. Nutrients 2 , $1231-1246$.

Uysal, S., Zengin, G., Aktumsek, A., Karatas, S., 2016. Chemical and biological approaches on nine fruit tree leaves collected from the Mediterranean region of Turkey. J. Funct. Foods 22, 518-532.

Vallverdú-Queralt, A., Jáuregui, O., Di Lecce, G., Andrés-Lacueva, C., Lamuela-Raventós, R.M., 2011. Screening of the polyphenol content of tomato-based products through accurate-mass spectrometry (HPLC-ESI-QTOF). Food Chem. 129, 877-883.

Van Hoyweghen, L., De Bosscher, K., Haegeman, G., Deforce, D., Heyerick, A., 2014. In vitro inhibition of the transcription factor NF-kB and cyclooxygenase by bamboo extracts. Phytother. Res. 28, 224-230.

Verardo, G., Duse, I., Callea, A., 2009. Analysis of underivatized oligosaccharides by liquid chromatography/electrospray ionization tandem mass spectrometry with post column addition of formic acid. Rapid Commun. Mass Spectrom. 23, 1607-1618.

Viciolle, E., Castilho, P., Rosado, C., 2012. In vitro and in vivo assessment of the effect of Laurus novocanariensis oil and essential oil in human skin. Int. J. Cosmet. Sci. 34, 546-550.

Vinha, A.F., Guido, L.F., Costa, A.S.G., Alves, R.C., Oliveira, M.B.P.P., 2015. Monomeric and oligomeric flavan-3-ols and antioxidant activity of leaves from different Laurus sp. Food Funct. 6, 1944-1949.

Waridel, P., Wolfender, J.-L., Ndjoko, K., Hobby, K.R., Major, H.J., Hostettmann, K., 2001. Evaluation of quadrupole time-of-flight tandem mass spectrometry and iontrap multiple-stage mass spectrometry for the differentiation of $C$-glycosidic flavonoid isomers. J. Chromatogr. A 926, 29-41.

Ye, M., Yan, Y., Guo, D.-A., 2005. Characterization of phenolic compounds in the Chinese herbal drug Tu-Si-Zi by liquid chromatography coupled to electrospray ionization mass spectrometry. Rapid Commun. Mass Spectrom. 19, 1469-1484. 